\title{
Long non-coding RNA NEAT1 regulates glioma cell proliferation and apoptosis by competitively binding to microRNA-324-5p and upregulating KCTD20 expression
}

\author{
JIALE ZHANG $^{1 *}$, YANGYANG LI ${ }^{2 *}$, YUQI LIU ${ }^{1 *}$, GUANGZHI XU ${ }^{1}$, \\ YUE HEI $^{1}$, XIAOMING LU ${ }^{2}$ and WEIPING LIU ${ }^{1}$ \\ ${ }^{1}$ Department of Neurosurgery, Xijing Hospital, Fourth Military Medical University, Xi'an, Shaanxi 710032; \\ ${ }^{2}$ Department of Neurosurgery, The First Affiliated Hospital of Nanjing Medical University, Nanjing, Jiangsu 210029, P.R. China
}

Received November 10, 2020; Accepted March 22, 2021

DOI: $10.3892 /$ or.2021.8076

\begin{abstract}
Previous studies have demonstrated that long non-coding RNAs (lncRNAs) serve a key role in the development and progression of several types of cancer, including glioma. The lncRNA nuclear paraspeckle assembly transcript 1 (NEAT1) contributes to cancer growth through its effects on cell proliferation, migration, invasion and drug resistance. However, the exact regulatory mechanisms via which NEAT1 acts in glioma are unclear. In the present study, the expression levels and function of NEAT1 in glioma tissues and cell lines were examined in vitro and in vivo. By reverse transcription-quantitative PCR and fluorescence in situ hybridization analysis, NEAT1 expression was upregulated in glioma tissues compared with in adjacent normal brain tissues, and elevated NEAT1 levels were associated with poor prognosis. Cell Counting Kit-8, colony formation, ethynyldeoxyuridine, flow cytometry and western blotting assays were performed to detect the effects of NEAT1 on cell biological behavior. Knockdown of NEAT1 in glioma cell lines was associated with cell cycle arrest at the $\mathrm{G}_{0} / \mathrm{G}_{1}$ phase, decreased proliferation and elevated apoptosis in vitro, and resulted in reduced tumor growth and increased survival in a mouse xenograft model of glioma. Using bioinformatics analysis, RNA
\end{abstract}

Correspondence to: Dr Weiping Liu, Department of Neurosurgery, Xijing Hospital, Fourth Military Medical University, 17 Changle West Road, Xi'an, Shaanxi 710032, P.R. China

E-mail: neurosurgeonliu@163.com

Dr Xiaoming Lu, Department of Neurosurgery, The First Affiliated Hospital of Nanjing Medical University, 300 Guangzhou Road, Nanjing, Jiangsu 210029, P.R. China

E-mail:1uxm923@126.com

${ }^{*}$ Contributed equally

Key words: glioma, nuclear paraspeckle assembly transcript 1, proliferation, apoptosis, microRNA-324-5p, potassium channel tetramerization protein domain containing 20 immunoprecipitation experiments and luciferase reporter assays, it was demonstrated that NEAT1 may competitively bind to microRNA (miR)-324-5p, thus blocking its interaction with target mRNAs. Potassium channel tetramerization protein domain containing 20 (KCTD20) was identified as a specific miR-324-5p target. Accordingly, the inhibition of NEAT1 resulted in the downregulation of KCTD20 through competitive binding with miR-324-5p, decreased cell proliferation and increased apoptosis. Concomitant NEAT1 knockdown and inhibition of miR-324-5p partially reversed the effects of NEAT1 knockdown on cell proliferation and apoptosis, and further regulated KCTD20 expression. Collectively, the present findings demonstrated that NEAT1 acted as a competing endogenous RNA for miR-324-5p, and identified the NEAT1/miR-324-5p/KCTD20 axis as a novel regulatory axis and a potential therapeutic target for human glioma.

\section{Introduction}

Glioma is the most prevalent and aggressive tumor of the central nervous system and accounts for $\sim 70 \%$ of malignant primary brain tumors (1). The past several decades have seen consistent improvements in diagnostic methods and treatment strategies for glioma, with standard treatment including surgical resection followed by radiotherapy and systemic temozolomide chemotherapy (2). However, the prognosis for patients with glioma remains poor, with an overall survival time of only $12-14$ months from diagnosis $(3,4)$. Thus, there is an urgent need to identify novel molecular targets associated with key processes involved in glioma development and progression, such as proliferation, apoptosis, differentiation, invasion and migration, which can facilitate the development of more effective treatment strategies.

Long non-coding RNAs (lncRNAs) are a subgroup of RNAs with transcripts of $>200$ nucleotides in length having little or no protein-coding potential $(5,6)$. It has been demonstrated that under normal physiological conditions, lncRNAs serve crucial roles in numerous fundamental organism- and cell-related processes, including development, genomic imprinting, homeostasis, embryonic stem cell pluripotency (7-9), and cell proliferation, metabolism, apoptosis, 
migration and differentiation (10-12). The regulatory mechanisms through which lncRNAs function are complex and not fully understood; however, they are known to occur through epigenetic, transcriptional and post-transcriptional mechanisms (13-15). A novel regulatory mechanism, in which lncRNAs act as competing endogenous RNAs (ceRNAs) by binding directly to complementary sequences in microRNAs (miRNAs/miRs) and competitively blocking miRNA interactions with their target mRNAs, has been identified $(16,17)$. Emerging evidence has demonstrated the importance of ceRNAs, also known as sponges, in cancer through the regulation of the expression levels of miRNAs and their target genes. For instance, the lncRNA metallothionein 1J, pseudogene acts as a ceRNA in gastric cancer to regulate the function of miR-92a-3p and the expression of its target gene F-box and WD repeat domain containing 7 (18). Similarly, the lncRNA tumor suppressor candidate 8 acts as a ceRNA for miR-190b-5p, which modulates the expression of myosin regulatory light chain interacting protein mRNA and inhibits breast cancer metastasis (19). Although these and other studies $(20,21)$ have established lncRNAs as core factors in the development and growth of cancer, little is known regarding the pathophysiological mechanisms of action of lncRNAs in glioma.

The lncRNA nuclear paraspeckle assembly transcript 1 (NEAT1), located on chromosome 11, has been reported to be a transcriptional regulator of numerous genes (22), and abnormal NEAT1 expression has been implicated in the promotion of tumorigenesis in a variety of human cancer types $(23,24)$. Among its key mechanisms of action in cancer, NEAT1 acts as a ceRNA for several tumor suppressor miRNAs (25). In non-small cell lung cancer (NSCLC), NEAT1 promotes growth under hypoxic conditions and regulates the Wnt/ $\beta$-catenin signaling pathway (26). The upregulation of NEAT1 has been demonstrated to facilitate pancreatic cancer progression through the negative regulation of miR-506-3p (27). To the best of our knowledge, it is not yet known whether NEAT1 is involved in glioma development and progression.

In the present study, the expression levels and function of NEAT1 in glioma tissues and cell lines were examined, and its mechanism of action was investigated in detail. NEAT1 was revealed to promote glioma cell proliferation in vitro and in vivo by functioning as a ceRNA for miR-324-5p, which, in turn, regulated the expression of potassium channel tetramerization protein domain containing 20 (KCTD20). The inhibition of NEAT1 and KCTD20 inhibited the proliferation of glioma cells, which was consistent with the results of miR-324-5p overexpression. Therefore, the results revealed a novel NEAT1/miR-324-5p/KCTD20 regulatory axis in glioma, and identified a potential target for the development of diagnostic, prognostic and/or therapeutic tools for this disease.

\section{Materials and methods}

Human tissue samples. All 43 human glioma tissues (age range, 26-71 years; mean age, 49 years), as well as their paired adjacent non-cancerous tissues, were obtained from patients who underwent surgical resection at The First Affiliated Hospital of Nanjing Medical University (Nanjing, China) between January 2013 and December 2016. The distance between the tumor and the matched normal adjacent tissue was $\sim 2 \mathrm{~cm}$. The inclusion criteria were as follows: i) Diagnosed with glioma; and ii) had not received pre-operative radiotherapy, chemotherapy or other adjuvant treatments before surgery. The exclusion criteria were as follows: i) Diagnosed with other diseases; and ii) failed to cooperate with researchers. Histological grade was classified by pathologists using the World Health Organization criteria (28). All tissue samples were collected during surgery, frozen immediately in liquid nitrogen, and stored at $-80^{\circ} \mathrm{C}$ for total RNA or protein extraction. The clinicopathological characteristics of the patients are summarized in Table I. The present study was approved by the Institutional Review Board and Ethics Committee of Nanjing Medical University (Nanjing, China) and Fourth Military Medical University (Xi'an, China), and written informed consent was obtained from all patients.

Cell culture. A total of six human glioblastoma (GBM) cell lines, including U87MG [GBM of unknown origin; American Type Culture Collection HTB-14; short-tandem repeat (STR) profiling was performed], LN229, H4, U251, U118 (derived from the U138MG astrocytoma cell line; American Type Culture Collection HTB-15; STR profiling was performed) and A172, were obtained from The Cell Bank of Type Culture Collection of The Chinese Academy of Sciences. The human 293T cell line was obtained from American Type Culture Collection. All cells were sustained in DMEM (HyClone; Cytiva) supplemented with 10\% FBS (HyClone; Cytiva), $100 \mathrm{U} / \mathrm{ml}$ penicillin and $100 \mathrm{ng} / \mathrm{ml}$ streptomycin. Normal human astrocytes (NHAs) were purchased from Lonza Group, Ltd. and cultured in the provided astrocyte growth media (Lonza Group, Ltd.) supplemented with $0.1 \%$ recombinant human epidermal growth factor, $0.25 \%$ insulin, $0.1 \%$ ascorbic acid, $0.1 \%$ GA-1000, 1\% L-glutamine (all Lonza Group, Ltd.) and $5 \%$ FBS. All cells were cultured at $37^{\circ} \mathrm{C}$ in a humidified atmosphere with $5 \% \mathrm{CO}_{2}$.

Total RNA extraction and reverse transcription-quantitative $P C R(R T-q P C R)$. Total RNA was extracted from glioma tissues or cultured cell lines using TRIzol ${ }^{\circledR}$ reagent (Invitrogen; Thermo Fisher Scientific, Inc.) according to the manufacturer's protocols. Single-stranded cDNA was synthesized using the Primerscript RT Master mix (Takara Bio, Inc.) according to the manufacturer's protocol. Subsequently, qPCR analysis was performed with SYBR Premix Ex Taq (Takara Bio, Inc.) according to the manufacturer's protocols. RT-qPCR was performed using an Applied Biosystems 7900 Sequence Detection system (Thermo Fisher Scientific, Inc.). The thermocycling conditions were as follows: $95^{\circ} \mathrm{C}$ for $3 \mathrm{~min}$, followed by 40 cycles at $95^{\circ} \mathrm{C}$ for $1 \mathrm{~min}$ and $57^{\circ} \mathrm{C}$ for $45 \mathrm{sec}$, and $72^{\circ} \mathrm{C}$ for 2 min. All primers used for RT and RT-qPCR were purchased from Guangzhou RiboBio Co., Ltd. and the sequences were as follows: NEAT1 forward, 5'-TGGCTAGCTCAGGGCTTC AG-3' and reverse, 5'-TCTCCTTGCCAAGCTTCCTTC-3'; miR-324-5p forward, 5'-CGCGGATCCGGGTGGATGTAA GGGATGAG-3' and reverse, 5'-CCGGAATTCTTGGGC TGATCCAGGAGAAG-3'; KCTD20 forward, 5'-CGGGAT CCATGAATGTTCACCGTGGCAG-3' and reverse, 5'-CGA ATTCCTAATCCTGAAAGTCGTTAGAAGC-3'; GAPDH forward, 5'-GACTCATGACCACAGTCCATGC-3' and reverse 
Table I. Association between the expression levels of NEAT1 and clinicopathological features of 43 patients with glioma.

\begin{tabular}{|c|c|c|c|c|}
\hline \multirow[b]{2}{*}{ Characteristics } & \multirow[b]{2}{*}{ Number } & \multicolumn{2}{|c|}{ NEAT1 expression } & \multirow[b]{2}{*}{ P-value } \\
\hline & & Low, n (n=22) & High, $n(n=21)$ & \\
\hline \multicolumn{5}{|l|}{ Age, years } \\
\hline$<45$ & 19 & 9 & 10 & 0.658 \\
\hline$\geq 45$ & 24 & 13 & 11 & \\
\hline \multicolumn{5}{|l|}{ Sex } \\
\hline Male & 23 & 10 & 13 & 0.346 \\
\hline Female & 20 & 12 & 8 & \\
\hline \multicolumn{5}{|l|}{ Tumor size, cm } \\
\hline$<5$ & 25 & 16 & 9 & $0.047^{\mathrm{a}}$ \\
\hline$\geq 5$ & 18 & 6 & 12 & \\
\hline \multicolumn{5}{|c|}{ Peritumoral brain edema, $\mathrm{cm}$} \\
\hline$<1$ & 22 & 9 & 13 & 0.169 \\
\hline$\geq 1$ & 21 & 13 & 8 & \\
\hline \multicolumn{5}{|l|}{ WHO grade } \\
\hline I-II & 27 & 17 & 10 & $0.044^{\mathrm{a}}$ \\
\hline III-IV & 16 & 5 & 11 & \\
\hline
\end{tabular}

${ }^{a} \mathrm{P}<0.05$ was considered to indicate a statistically significant difference. NEAT1, nuclear paraspeckle assembly transcript 1 ; WHO, World Health Organization.

5'-AGAGGCAGGGATGATGTTCTG-3'; and U6 forward, 5'-CTCGCTTCGGCAGCACA-3' and reverse, 5'-AACGCT TCACGAATTTGCGT-3'. The relative expression levels of NEAT1 and KCTD20 were normalized to those of GAPDH, while U6 was used as an internal control for miRNA. The expression levels of NEAT1, miR-324-5p and KCTD20 were calculated using $2^{-\Delta \Delta \mathrm{Cq}}$ analysis (29).

Cell transfection. The corresponding negative controls (NCs) and NEAT1 [small interfering RNA (si/siRNA)NEAT1], KCTD20 (siKCTD20), miR-324-5p inhibitor and miR-324-5p mimics were synthesized by Shanghai GenePharma Co., Ltd. The sequences were as follows: si control (Ctrl), 5'-CCCACC AGUUUGAGACUCCACAAAU-3'; siNEAT1, 5'-GGTCTG TGTGGAAGGAGGAAGGCAG-3'; siKCTD20, 5'-GGGAGG AAUAUUCCCAAAUTT-3'; miR-324-5p mimic, 5'-CGC AUCCCCUAGGGCAUUGGUGU-3'; miR-324-5p inhibitor, 5'-ACACCA AUGCCCUAGGGGAUGCG-3'; mimic NC (miR-NC), 5'-UUCUCCGAACGUGUCACGU-3'; and inhibitor NC (Anti-Ctrl), 5'-CAGUACUUUUGUGUAGUACAA-3'. When cells reached a confluence of $80 \%$, cells were transfected with siRNAs $(100 \mathrm{nM})$, mimics $(100 \mathrm{nM})$ or miRNA inhibitors $(100 \mathrm{nM})$ using Lipofectamine ${ }^{\circledR} 2000$ transfection reagent (Invitrogen; Thermo Fisher Scientific, Inc.) according to the manufacturer's protocol. The cells were then cultured using DMEM containing 10\% FBS, $100 \mathrm{U} / 1$ penicillin and $100 \mathrm{mg} / \mathrm{l}$ streptomycin at $5 \% \mathrm{CO}_{2}$ and $37^{\circ} \mathrm{C}$ for $48 \mathrm{~h}$. The transfected cells were harvested $48 \mathrm{~h}$ post transfection and used for subsequent experiments. To overexpress lncRNA NEAT1 in GBM cells, $3 \mu \mathrm{g}$ pcDNA3.1-NEAT1 (pcDNA-NEAT1) and $3 \mu \mathrm{g}$ negative control (empty Vector) (both Shanghai GenePharma Co., Ltd.) were transfected into cells using Lipofectamine ${ }^{\circledR} 2000$
(Invitrogen; Thermo Fisher Scientific, Inc.) according to the manufacturer's protocol. The cells were incubated in complete medium supplemented with $10 \% \mathrm{FBS}, 100 \mathrm{U} / 1$ penicillin and $100 \mathrm{mg} / \mathrm{l}$ streptomycin for $48 \mathrm{~h}$ at $37^{\circ} \mathrm{C}$ with $5 \% \mathrm{CO}_{2}$, and subsequent experiments were performed after $48 \mathrm{~h}$ of transfection. For stable transfection, the shRNA lentiviral vectors targeting NEAT1 (shNEAT1, 5'-CATGGACCGTGGTTTGTTACT-3') and KCTD20 (shKCTD20, 5'-GCTTCCAAAGTGGGAATA AAC-3') were synthesized by Shanghai GenePharma Co., Ltd. Lentiviruses were produced using a second-generation lentiviral system in 293T cells. 293T cells in the logarithmic growth phase were harvested and re-suspended in antibiotic-free and serum-free medium. Subsequently, cells $\left(5 \times 10^{5}\right.$ cells $\left./ \mathrm{ml}\right)$ were cultured in DMEM (HyClone; Cytiva) supplemented with 10\% FBS (HyClone; Cytiva), $100 \mathrm{U} / \mathrm{ml}$ penicillin and $100 \mathrm{ng} / \mathrm{ml}$ streptomycin in a 6 -well plates at $37^{\circ} \mathrm{C}$ with $5 \%$ $\mathrm{CO}_{2}$ until the cells reached $80 \%$ confluence. Briefly, shNEAT1 or shKCTD20 was inserted into the pLKO.1 vector (BioSettia, Inc.) and transfected into 293T cells (American Type Culture Collection) together with $0.75 \mu \mathrm{g}$ psPAX2 (BioSettia, Inc.) and $0.25 \mu \mathrm{g}$ pMD2.G envelope plasmids (BioSettia, Inc.; lentiviral plasmid: Packaging vector: Envelope $=4: 3: 1)$ using Lipofectamine ${ }^{\circledR} 2000$ (Invitrogen; Thermo Fisher Scientific, Inc.) to produce an shRNA-containing lentivirus. The media containing lentiviral particles were harvested at $48 \mathrm{~h}$ after transfection. Next, cells in the media were removed using a $0.45-\mu \mathrm{m}$ filter (EMD Millipore) and viral particles were collected. Subsequently, U251 cells were transduced with shNC, shNEAT1 or shKCTD20 lentiviruses (multiplicity of infection, 10) for $24 \mathrm{~h}$ at $37^{\circ} \mathrm{C}$ with $5 \% \mathrm{CO}_{2}$ in the presence of $5 \mu \mathrm{g} / \mathrm{ml}$ polybrene (Shanghai GenePharma Co., Ltd.). After $48 \mathrm{~h}$ of lentivirus infection, cells were selected using $5 \mathrm{mg} / \mathrm{ml}$ 
puromycin (Sigma-Aldrich; Merck KGaA) and maintained in puromycin-containing medium $(5 \mathrm{mg} / \mathrm{ml})$ for 14 days to establish stably transduced cell lines.

Cell Counting Kit-8 (CCK-8) assay. At $48 \mathrm{~h}$ after transfection, cells were seeded at 2,000 cells per well in 96-well plates and cultured. First, the cell proliferation rate was detected at the indicated time points $(0,24,48,72$ and $96 \mathrm{~h})$ using a CCK-8 assay (Dojindo Molecular Technologies, Inc.) according to the manufacturer's protocols. After $1 \mathrm{~h}$ of incubation with CCK-8 at $37^{\circ} \mathrm{C}$, the absorbance (optical density value) at a wavelength of $450 \mathrm{~nm}$ was detected and used to calculate cell proliferation.

Colony formation assay. The experimental procedures were performed according to the method described in our previous study (30). Briefly, cells were harvested $48 \mathrm{~h}$ after transfection and then seeded into a 6 -well plate (200 cells/well). Following culture for $\sim 2$ weeks until colony formation was observed, visible colonies were fixed with $100 \%$ methanol for $20 \mathrm{~min}$ at room temperature and stained with $0.1 \%$ crystal violet (Sigma-Aldrich; Merck KGaA) for $15 \mathrm{~min}$ at room temperature. The colonies were counted using an inverted light microscope (Olympus X71; Olympus Corporation). The numbers of colonies were then counted and measured using ImageJ software (version 1.51; National Institutes of Health). Colony formation efficiency was calculated as the number of colonies/plated cells x100\%.

Ethynyldeoxyuridine (EdU) assay. For the EdU proliferation assay, the Cell-Light EdU labeling detection kit was purchased from Thermo Fisher Scientific, Inc. As aforementioned, U251 and LN229 cells in 96-well plates $\left(2 \times 10^{3}\right.$ cells/well) were transfected with plasmid DNA or siRNA for $48 \mathrm{~h}$. Then, $10 \mu \mathrm{M}$ EdU was added to the 96-well plates, and they were incubated for $24 \mathrm{~h}$ at $37^{\circ} \mathrm{C}$ with $5 \% \mathrm{CO}_{2}$. The medium was then discarded and cells were washed twice with PBS at room temperature for 3 min each time. The cells were fixed with $4 \%$ paraformaldehyde in PBS for $25 \mathrm{~min}$ at room temperature and 0.5\% Triton X-100 in PBS for 20 min at room temperature. Then, cells were stained with the Alexa-Fluor 594 reaction cocktail for EdU. After removal of the reaction cocktail, cells were washed with 3\% BSA (Thermo Fisher Scientific, Inc.) in PBS at room temperature. Mounting medium with DAPI ( $2 \mu \mathrm{g} / \mathrm{ml}$; cat. no. ab104139; Abcam) was used to label the cell nuclei for $15 \mathrm{~min}$ at room temperature, the cells were then washed with PBS at room temperature three times (30 sec each time). A total of five visual fields were selected randomly using a fluorescence microscope (magnification, x200; Leica Microsystems $\mathrm{GmbH}$ ). The EdU-positive cells and DAPI stained cells (total cells) were counted. Cell proliferation rate $=$ number of proliferative cells/number of total cells $\times 100 \%$.

Flow cytometry. Cell cycle analysis was performed as previously described (31). For cell cycle analysis, U251 and LN229 cells were transfected with the plasmids or siRNA for $48 \mathrm{~h}$ and then collected by centrifugation for $5 \mathrm{~min}$ at $1,500 \mathrm{x} \mathrm{g}$ at room temperature. Subsequently, cells were washed twice with PBS and fixed with $75 \%$ ice-cold ethanol at $4^{\circ} \mathrm{C}$ for $24 \mathrm{~h}$. The collected cells were re-suspended in PBS containing $25 \mathrm{mg} / \mathrm{ml} \mathrm{PI}, 0.1 \%$ Triton and $10 \mathrm{mg} / \mathrm{ml}$ RNase [Hangzhou
Multi Sciences (Lianke) Biotech Co., Ltd.] and incubated for $30 \mathrm{~min}$ in the dark, before being analyzed by flow cytometry. The apoptotic cells were stained with Annexin V-FITC/PI, the cells were analyzed with a Gallios flow cytometer (Beckman Coulter, Inc.) equipped with Kaluza 2.1.1 software (Beckman Coulter, Inc.). Cells were divided into dead cells (B1), late apoptotic cells (B2), negative control normal cells (B3) and early apoptotic cells (B4) according to the different state of the cell. In each experiment, the total percentage of early and late apoptotic cells was compared with the controls.

Western blotting and antibodies. Briefly, cultured cells were lysed on ice for 30 min in RIPA buffer (Nanjing KeyGen Biotech Co., Ltd.). Subsequently, total protein extraction of different transfected U251 and LN229 cells was evaluated using a BCA Protein Assay kit (Pierce; Thermo Fisher Scientific). Protein (30 $\mu$ g per lane) was loaded, subjected to 10\% SDS-PAGE and then transferred onto PVDF membranes (EMD Millipore) for western blotting. After blocking the membranes with $5 \%$ non-fat for $2 \mathrm{~h}$ at room temperature, the membranes were probed with antibodies against CDK4 (dilution, 1:1,000; cat. no. ab108357; Abcam), cyclin D1 (dilution, 1:1,000; cat. no. ab40754; Abcam), Bcl-2 (dilution, 1:1,000; cat. no. ab59348; Abcam), Bax (dilution, 1:1,000; cat. no. ab32503; Abcam), KCTD20 (dilution, 1:500; cat. no. ab122094; Abcam), NIPBL (dilution, 1:500; cat. no. ab225908; Abcam), ELAVL1 (dilution, 1:1,000; cat. no. ab200342; Abcam), KLF3 (dilution, 1:500; cat. no. ab154531; Abcam), MMP19 (dilution, 1:500; cat. no. ab53146; Abcam), ZFX (dilution, 1:500; cat. no. 5419; Cell Signaling Technology, Inc.) and GAPDH (dilution, 1:1,000; cat. no. AF5009; Beyotime Institute of Biotechnology) at $4^{\circ} \mathrm{C}$ overnight. Membranes were then incubated with corresponding secondary antibodies goat anti-mouse (dilution, 1:1,000; cat. no. A0216; Beyotime Institute of Biotechnology) and goat anti-rabbit (dilution, 1:1,000; cat. no. A0208; Beyotime Institute of Biotechnology) for $1 \mathrm{~h}$ at room temperature, and processed using ECL Western Blot Detection reagents (EMD Millipore). An ECL detection system (Thermo Fisher Scientific, Inc.) was used to visualize the protein. GAPDH was used as an internal control. Signals were examined by densitometric scans using ImageJ software (version 1.51; National Institutes of Health).

RNA immunoprecipitation (RIP). For the RIP assay, pSL-MS2-12X (Addgene, Inc.) was double digested using Bam HI and XhoI, and the MS2-12X fragment was inserted to the pcDNA3.1-NEAT1 vector to form pSL-MS2-NEAT1. Next, U251 and LN229 cells were co-transfected with pMS2-GFP and pSL-MS2-NETA1 or pSL-MS2-12x vectors (Addgene, Inc.). After $48 \mathrm{~h}$, the cells were used for the RIP assay with a green fluorescent protein antibody (Roche Diagnostics). The RIP assay was performed using the Magna RIP RNA-Binding Protein Immunoprecipitation kit (EMD Millipore) according to the manufacturer's protocols. Briefly, U251 and LN229 cells were collected by centrifugation at $800 \mathrm{x} \mathrm{g}$ for $5 \mathrm{~min}$ at $4^{\circ} \mathrm{C}$ and then lysed using RIP lysis buffer (EMD Millipore). One RIP reaction required $100 \mu \mathrm{l}$ of cell lysate from $\sim 2.0 \times 10^{7}$ cells. Subsequently, cell lysates were incubated with $50 \mu \mathrm{l} \mathrm{A} / \mathrm{G}$ magnetic beads conjugated with human anti-argonaute RISC catalytic component 2 (Ago2) antibody (dilution, 1:150; cat. no. ab32381; Abcam) 
for $30 \mathrm{~min}$ at room temperature, while normal rabbit $\mathrm{IgG}$ (dilution, 1:150; cat. no. 12-370; EMD Millipore) served as a negative control. Next, the compounds of antibody and magnetic beads in $900 \mu \mathrm{l}$ RIP Immunoprecipitation Buffer (EMD Millipore) were co-incubated overnight at $4^{\circ} \mathrm{C}$ with cell lysates supernatants $(100 \mu \mathrm{l})$ after centrifugation at $13,000 \mathrm{x} \mathrm{g}$ for $10 \mathrm{~min}$ at $4^{\circ} \mathrm{C}$. Next, the beads $(50 \mu \mathrm{l})$ were collected with a magnetic separator, washed three times with cold RIP Wash Buffer (EMD Millipore). Then, the complexes were incubated with $0.1 \% \mathrm{SDS} / 0.5 \mathrm{mg} / \mathrm{ml}$ proteinase K (EMD Millipore) for $30 \mathrm{~min}$ at $55^{\circ} \mathrm{C}$ to remove proteins and immunoprecipitated RNA was isolated. NEAT1 and miR-324-5p expression was assessed by RT-qPCR, as aforementioned.

Luciferase reporter assay. The complementary DNA fragment containing the wild-type (WT) or mutant (MUT) sequences of NEAT1 or KCTD20 were subcloned downstream of the luciferase gene within the pGL3-luciferase reporter plasmid vectors (Promega Corporation). Plasmids containing the sequences of miR-324-5p mimic (5'-CGCAUCCCCUAGGGC AUUGGUGU-3') and miR-NC (5'-UUCUCCGAACGUGUC ACGU-3') were purchased from Shanghai GenePharma Co., Ltd. Briefly, cells were seeded into 24-well plates and cultured overnight. The cells were then co-transfected with WT- or MUT-NEAT1 and the 3' untranslated region (UTR) of the KCTD20 fragment, as well as equal amounts of miR-NC and miR-324-5p using Lipofectamine ${ }^{\circledR} 2000$ (Invitrogen; Thermo Fisher Scientific, Inc.) according to the manufacturer's protocols. At $48 \mathrm{~h}$ after transfection, the relative luciferase activity in the cells was measured using a Dual Luciferase Reporter Assay System (Promega Corporation). The relative luciferase activity was normalized to the Renilla luciferase activity. All experiments were performed in triplicate.

Orthotopic xenograft studies. A total of 24 male immunodeficient nude mice $(\mathrm{n}=6$ for each group; age, 6 weeks; weight, $\sim 20 \mathrm{~g})$ were purchased from Shanghai Laboratory Animal Research Center. The mice were maintained under specific pathogen-free conditions in a temperature-controlled room $\left(\sim 20^{\circ} \mathrm{C}\right.$; humidity, $\left.20 \%\right)$, with a 12 -h light/dark cycle, and with ad libitum access to commercially available mouse food and sterilized water. To examine tumor growth in the orthotopic xenograft model, a total of $100 \mu$ l of PBS containing U251 cells $\left(2.5 \times 10^{5}\right)$ were stably transfected with shNEAT1 or shKCTD20 and the corresponding negative control (shNC) was injected intracranially into the striatum of NOD/SCID mice by a stereotactic device (coordinates, $2 \mathrm{~mm}$ behind the anterior fontanel, $2 \mathrm{~mm}$ lateral to the sagittal suture, at a $3-\mathrm{mm}$ depth from the dura). A bioluminescence imaging system (IVIS Spectrum; PerkinElmer, Inc.) was used to confirm tumor formation and measure tumor growth weekly. At 7, 14, 21 and 28 days after cell implantation, the mice were injected intraperitoneally with D-luciferin at $50 \mathrm{mg} / \mathrm{ml}$ and then subjected to in vivo bioluminescence imaging to visualize tumor growth for 10-120 sec. The whole experiment lasted $\sim 2$ months. During this process, according to development of neurological signs (hunching, weight loss, rough coat), the moribund mice were anesthetized by an intraperitoneal injection of $10 \%$ chloral hydrate ( $400 \mathrm{mg} / \mathrm{kg}$ body weight). The mice showed no signs of peritonitis, pain or other discomfort, and were then euthanized by means of cervical dislocation. Their brains were harvested, cut into sections, paraffin-embedded and stained with H\&E to confirm the presence of tumors, and subjected to immunohistochemistry (IHC). For H\&E staining, brain tissues were fixed with $10 \%$ formaldehyde at room temperature for $24 \mathrm{~h}$. Subsequently, paraffin-embedded specimens were cut into 5- $\mu \mathrm{m}$ sections and dewaxed with xylene and cleared with a series of changing alcohol concentrations. The samples were washed three times in PBS (5 min each time) at room temperature, and then stained with hematoxylin (Sigma-Aldrich; Merck KGaA) for $5 \mathrm{~min}$ at room temperature. Sections were stained with eosin (Sigma-Aldrich; Merck $\mathrm{KGaA}$ ) for $2 \mathrm{~min}$ at room temperature to observe the clarity of the nucleus and cytoplasm under a light microscope (Leica Microsystems $\mathrm{GmbH}$ ). The images were assessed using Image-Pro Plus 6.0 (Media Cybernetics, Inc.). For IHC, tumor tissues were fixed with $10 \%$ formaldehyde for $24 \mathrm{~h}$ at room temperature and sectioned into 5- $\mu$ m-thick slides, followed by incubation with citrate buffer (Thermo Fisher Scientific, Inc.) under high pressure to repair the antigen for $3 \mathrm{~min}$. The slides were incubated with $0.3 \% \mathrm{H}_{2} \mathrm{O}_{2}$ for $20 \mathrm{~min}$ at room temperature to quench the endogenous peroxidase. Next, blocking reagent (3\% BSA; Thermo Fisher Scientific, Inc.) was used to reduce the non-specific binding of antibodies at room temperature for $1 \mathrm{~h}$. Primary antibodies against Ki-67 (dilution, 1:200; cat. no. ab16667; Abcam) and KCTD20 (dilution, 1:100; cat. no. ab122094; Abcam) were used for incubation at $4^{\circ} \mathrm{C}$ overnight. After washing with PBS, the slides were incubated with biotinylated secondary antibody (dilution 1:500; cat. no. ab207995; Abcam) for $1 \mathrm{~h}$ at room temperature. The slides were incubated with HRP-conjugated streptavidin for $40 \mathrm{~min}$ at room temperature and then the DAB chromogen (Promega Corporation) was used for visualization. Slides were imaged under a light microscope (Leica Microsystems $\mathrm{GmbH}$ ). The maximum diameter of the tumor was $\sim 1.1 \mathrm{~cm}$ and the tumor volume $0.56 \mathrm{~cm}^{3}$. Tumor volume was calculated using the following formula: Tumor volume=length $\mathrm{x}$ width ${ }^{2} / 2$. In addition, the overall survival of the mice was monitored during the experimental period. All animal experimental procedures were approved by the Fourth Military Medical University Institutional Committee for Animal Research (Xi'an, China), and were in accordance with the Animal Management Rule of the Chinese Ministry of Health (document 55, 2001) (32).

Fluorescence in situ hybridization (FISH). 1ncRNA NEAT1 expression in gliomatissues and normal samples was examined by FISH. Briefly, tissues were fixed with $10 \%$ formaldehyde at room temperature for $2 \mathrm{~h}$ and embedded in paraffin, and then cut into 5- $\mu \mathrm{m}$ tissue slices. The NEAT1 sequence (5'-CGA GAAACGCACAAGAAGGCAGGCAAACAG-3') was synthesized by Wuhan Servicebio Technology Co., Ltd. (probe type, oligonucleotide; template source, human), and marked with 5' digoxigenin (DIG; cat. no. GDP1070; Wuhan Servicebio Technology Co., Ltd.) at $37^{\circ} \mathrm{C}$ for $16 \mathrm{~h}$. Treated sections were digested with Proteinase K $(20 \mu \mathrm{g} / \mathrm{ml}$; Sigma-Aldrich; Merck $\mathrm{KGaA}$ ) for $3 \mathrm{~min}$ at $37^{\circ} \mathrm{C}$, washed three times for $5 \mathrm{~min}$ each with deionized water and PBS at room temperature. Next, the sections were post-fixed in $10 \%$ paraformaldehyde fixative in PBS for $20 \mathrm{~min}$ at room temperature and washed three times (5 min each time) with PBS. And then tissue samples were 
incubated with pre-hybridization solution (Wuhan Servicebio Technology Co., Ltd.) in an incubator at $37^{\circ} \mathrm{C}$ for $1 \mathrm{~h}$. Next, after removing the pre-hybridization solution, the hybridization solution (containing $6 \mathrm{ng} / \mu \mathrm{l}$ NEAT probe; Wuhan Servicebio Technology Co., Ltd.) was added onto the slides, followed by incubation at $37^{\circ} \mathrm{C}$ overnight. Tissue sections were washed with preheated $2 \mathrm{X}$ sodium citrate (SSC) for $10 \mathrm{~min}$, $1 \mathrm{X}$ SSC (twice for $5 \mathrm{~min}$ each) and $0.5 \mathrm{X} \mathrm{SSC}$ in sequence for $10 \mathrm{~min}$ at $37^{\circ} \mathrm{C}$ to remove non-specific and repetitive RNA hybridization. Subsequently, sections were blocked with 3\% BSA (Thermo Fisher Scientific, Inc.) at room temperature for $30 \mathrm{~min}$, followed by incubation with anti-DIG-488 antibody (dilution, 1:300; Jackson ImmunoResearch Laboratories, Inc.) for $50 \mathrm{~min}$ and washed three times ( 3 min each) with PBS at $37^{\circ} \mathrm{C}$. Afterwards, the FITC-TSA reagent (dilution, 1:3,000; Wuhan Servicebio Technology Co., Ltd.) was used for incubation for $5 \mathrm{~min}$ at room temperature in the dark, followed by washing with PBS three times for $5 \mathrm{~min}$ each, and incubation with aqueous fluoroshield mounting medium with DAPI $(2 \mu \mathrm{g} / \mathrm{ml}$; cat. no. ab104139; Abcam) for nuclei staining in the dark for $15 \mathrm{~min}$ at room temperature. Finally, the slides were removed from the plate and fixed with 50\% glycerol in PBS at room temperature for $30 \mathrm{~min}$. Sections were then examined with a Zeiss LSM 700 confocal microscope (Zeiss AG). The images were analyzed using ImageJ software (version 1.51; National Institutes of Health).

Bioinformatics analysis. All statistical analysis was performed using GraphPad Prism 5.0 (GraphPad Software, Inc.). Gene expression profiles for patients with glioma [low grade glioma (LGG) and high-grade glioma (HGG; GBM)] were obtained from The Cancer Genome Atlas (TCGA; http://tcga-data.nci.nih.gov/tcga/) (33) and clinical data for overall survival were also download from TCGA (34). Gliomas were categorized as LGG [World Health Organization (WHO) Grade I-II] and HGG (WHO Grade III-IV) (35). China Glioma Genome Atlas (CGGA; http://www.cgga.org. cn/) (36) and Gene Expression Omnibus (dataset accession no. GSE16011; https://www.ncbi.nlm.nih.gov/geo/query/acc. cgi?acc=GSE16011) (37) were used to select differentially expressed lncRNAs, miRNAs and target genes. Furthermore, StarbaseV2.0 (http://starbase.sysu.edu.cn) and lncRNASNP2 (http://bioinfo.life.hust.edu.cn/lncRNASNP/\#!/) (38) were used to predict the potentially targeting relationship between IncRNAs and miRNAs. Based on the dual luciferase reporter assay results, lncRNASNP2 was used to predict the binding site between NEAT1 and miR-324-5p. Three online prediction tools, including Targetscan 7.1 (http://www.targetscan. org/), miRDB (http://mirdb.org) (39) and miRWalk 2.0 (http://mirwalk.umm.uni-heidelberg.de), were used to search for specific miRNA-mRNA relationships. The different results of online data prediction were analyzed and used to draw Venn diagrams using the Venn online analysis software (http://bioinformatics.psb.ugent.be/webtools/Venn/) (40). In addition, putative binding sites between miR-324-5p and KCTD20 from Targetscan were used for miRNA target validation analysis.

Statistical analysis. All experiments were performed in triplicate and data are presented as the mean \pm SD. All statistical analysis was carried out using GraphPad Prism 5.0 (GraphPad Software, Inc.). Comparisons between tumor and adjacent normal tissues were performed using a paired Student's t-test and the experimental and control groups were compared using an unpaired Student's t-test. One-way ANOVA was used to compare multiple different groups followed by Bonferroni's test. Survival curves were analyzed using Kaplan-Meier analysis, and significance was determined using the log-rank test. For TCGA Kaplan-Meier analysis, the median expression level was used as the cut-off value $(n=314>$ median; $n=314$ $\leq$ median). The $\chi^{2}$ test was used to analyze the association between NEAT1 and the clinicopathologic characteristics of patients. The correlation between NEAT1, miR-324-5p and KCTD20 expression was determined by Spearman's rank correlation analysis. $\mathrm{P}<0.05$ was considered to indicate a statistically significant difference.

\section{Results}

NEAT1 expression is upregulated in glioma tissues and is associated with poor prognosis. To evaluate aberrantly expressed lncRNAs in glioma and to identify those that may be involved in tumorigenesis, the glioma datasets LGG and HGG (GBM) from TCGA was analyzed. Among the dysregulated lncRNAs detected, NEAT1 expression was significantly upregulated in high-grade gliomas (HGG; $n=172$ ) compared with in low-grade gliomas (LGG; n=530; Fig. 1A). In addition, RT-qPCR analysis of NEAT1 levels in 43 matched pairs of clinical specimens revealed significantly elevated NEAT1 expression in glioma tissues compared with adjacent normal brain tissues ( $n=43$; Fig. 1B). Additionally, NEAT1 expression was examined in NHAs and a panel of six human glioma cell lines (U87MG, LN229, H4, U251, U118 and A172), and significantly higher NEAT1 expression was detected in all six tumor cell lines, particularly U251 and LN229 cells, compared with in NHAs (Fig. 1C). Furthermore, FISH and IHC demonstrated that the expression levels of NEAT1 and Ki-67, a proliferation marker, were upregulated in glioma sections compared with in normal brain sections (Fig. 1D), which was consistent with the RT-qPCR results (Fig. 1B).

To assess the effect of NEAT1 upregulation on the prognosis of patients with glioma, Kaplan-Meier survival analysis was conducted using the median NEAT1 expression level as the cut-off value for dichotomization of patients from the dataset obtained from TCGA. It was revealed that high NEAT1 expression was significantly associated with a shorter overall survival (Fig. 1E). Furthermore, 43 patients were divided into two groups with high or low NEAT1 expression, using the median expression level as the cut-off value $(n=22>$ median; $\mathrm{n}=22 \leq$ median). The results demonstrated that high NEAT1 expression was significantly associated with a larger tumor size $(\mathrm{P}=0.047)$ and advanced World Health Organization glioma stage $(\mathrm{P}=0.044$; Table $\mathrm{I})$. In combination, these data suggested that NEAT1 expression was significantly upregulated in glioma and could potentially serve as a prognostic marker for patients with glioma.

NEAT1 promotes the proliferation of glioma cells and induces apoptosis in vitro. To explore the potential mechanisms via which elevated NEAT1 expression may influence glioma cell biology, gain- and loss-of-function experiments with U251 and LN229 cell lines, which had the highest NEAT1 expression 
A

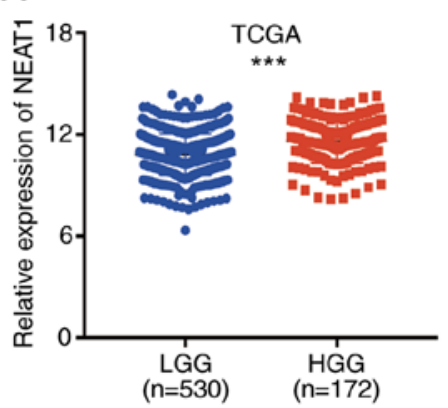

B

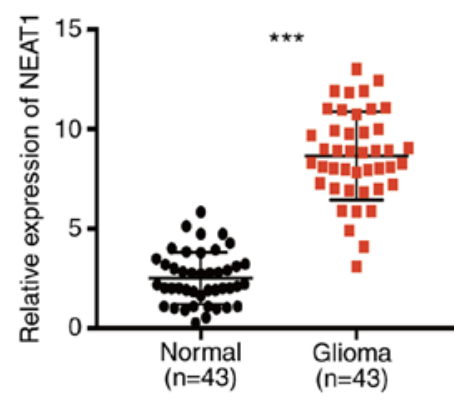

C

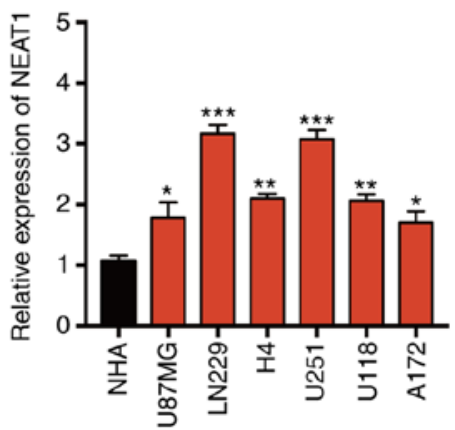

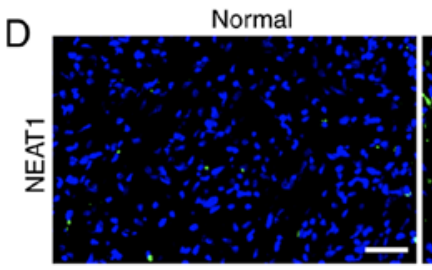
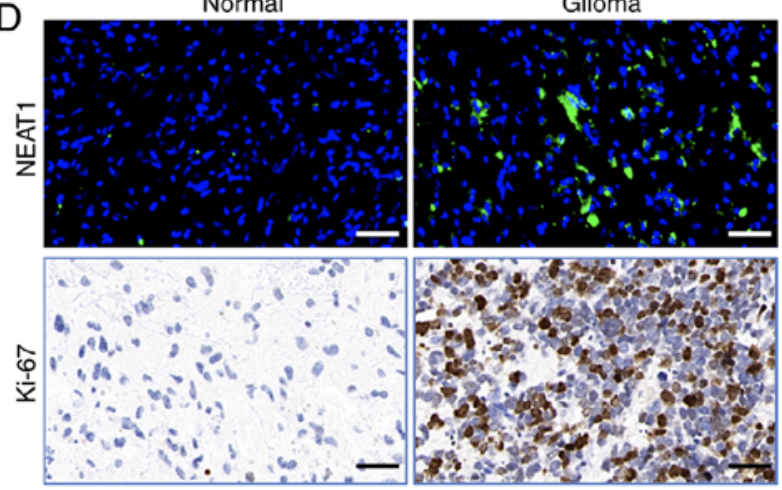

E

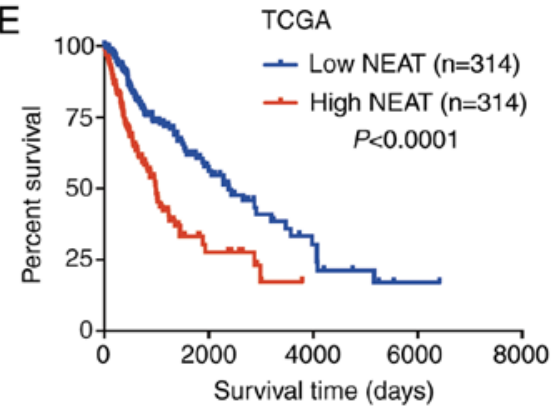

Figure 1. Expression levels of long non-coding RNA NEAT1 in glioma tissues and cell lines. (A) NEAT1 expression in LGG and HGG tissues in a TCGA dataset. (B) RT-qPCR of NEAT1 expression in 43 pairs of glioma tissues and adjacent non-tumor tissues. Data were normalized to GAPDH mRNA expression. (C) RT-qPCR of NEAT1 expression in NHAs and six glioma cell lines (U87MG, LN229, H4, U251, U118 and A172). (D) Fluorescence in situ hybridization of NEAT1 expression (upper panels) and immunohistochemical staining of Ki-67 expression (lower panels) in glioma tissues and adjacent non-tumor tissues. Scale bar, $100 \mu \mathrm{m}$. (E) Kaplan-Meier survival analysis of patients with glioma stratified by high ( $\mathrm{n}=314)$ and low (n=314) tumor expression levels of NEAT1. Data were obtained from TCGA. The experiments were performed in triplicate and data are presented as the mean $\pm \mathrm{SD}$. ${ }^{*} \mathrm{P}<0.05$, ${ }^{* *} \mathrm{P}<0.01$ and ${ }^{* * *} \mathrm{P}<0.001$ vs. normal astrocytes (NHA) or as indicated. NEAT1, nuclear paraspeckle assembly transcript 1; NHAs, normal human astrocytes; LGG, low-grade glioma; HGG, high-grade glioma; RT-qPCR, reverse transcription quantitative PCR; TCGA, The Cancer Genome Atlas.

levels (Fig. 1C), were performed by transfecting them with a control sequence (siCtrl) or NEAT1-specific siRNA (siNEAT1), or with a NEAT1 overexpression vector (pcDNA 3.1-NEAT1) or empty vector. RT-qPCR was performed $48 \mathrm{~h}$ after transfection and demonstrated that NEAT1 siRNA significantly reduced NEAT1 expression in U251 and LN229 cells, while transfection of pcDNA-NEAT1 increased NEAT1 expression (Fig. 2A).

To analyze the effects of NEAT1 regulation on glioma cell function, the proliferation of transfected cells was assessed using CCK-8, EdU staining and colony formation assays. The results revealed significantly reduced proliferation in cells expressing siNEAT1 compared in cells transfected with siCtrl (Fig. 2B, D and F), as reflected in all three assays. Conversely, the overexpression of NEAT1 promoted cell proliferation in both U251 and LN229 cells in all three assays (Fig. 2C, E and G). Collectively, these results suggested that NEAT1 may act as an oncogene in glioma.

Knockdown of NEAT1 induces cell cycle arrest and apoptosis in glioma cells in vitro and suppresses gliomagenesis in vivo. To determine the potential mechanism through which NEAT1 may promote glioma cell proliferation, the effects of knockdown or overexpression of NEAT1 on the cell cycle distribution and apoptotic rate of U251 and LN229 cells were analyzed using flow cytometry. Downregulation of NEAT1 expression resulted in a marked accumulation of $\mathrm{U} 251$ and LN229 cells at the $G_{0} / G_{1}$ phase of the cell cycle, with a concomitant decrease in the proportion of cells at the $\mathrm{S}$ phase (Fig. 3A). Conversely, an increase in cell cycle progression of $\mathrm{G}_{1}-\mathrm{S}$ transition was observed in NEAT1-overexpressing U251 (S phase increased from 21.1 to $34.6 \%$ ) and LN229 cells (S phase increased from 23.2 to $41.4 \%$; Fig. 3B). Consistent with these results, the proportion of cells undergoing apoptosis was increased significantly following knockdown of NEAT1 and decreased following overexpression of NEAT1 (Fig. 3C and D). These results were supported by the analysis of the expression levels of the cell cycle-related proteins CDK-4 and cyclin D1, the pro-apoptotic protein Bax and the anti-apoptotic protein Bcl-2. Western blotting demonstrated that the protein expression levels of CDK-4, cyclin D1, Bcl-2 and Bax were upregulated or downregulated in a manner consistent with the functional effects of knockdown and overexpression of NEAT1 on cell cycle progression and apoptosis. As shown in Fig. 3E, knockdown of NEAT1 was associated with decreased expression levels of cycle-related proteins (CDK-4 and cyclin D1) and apoptosis-related protein $\mathrm{Bcl}-2$ but the expression levels of apoptosis-related protein Bax were increased. By contrast, the overexpression of NEAT1 inhibited the expression of Bax and promoted the expression of CDK-4, cyclin D1 and Bcl-2 (Fig. 3E).

Subsequently, the present study examined whether the role of NEAT1 in the promotion of glioma cell proliferation in vitro could also be observed in vivo. U251 cell lines stably 

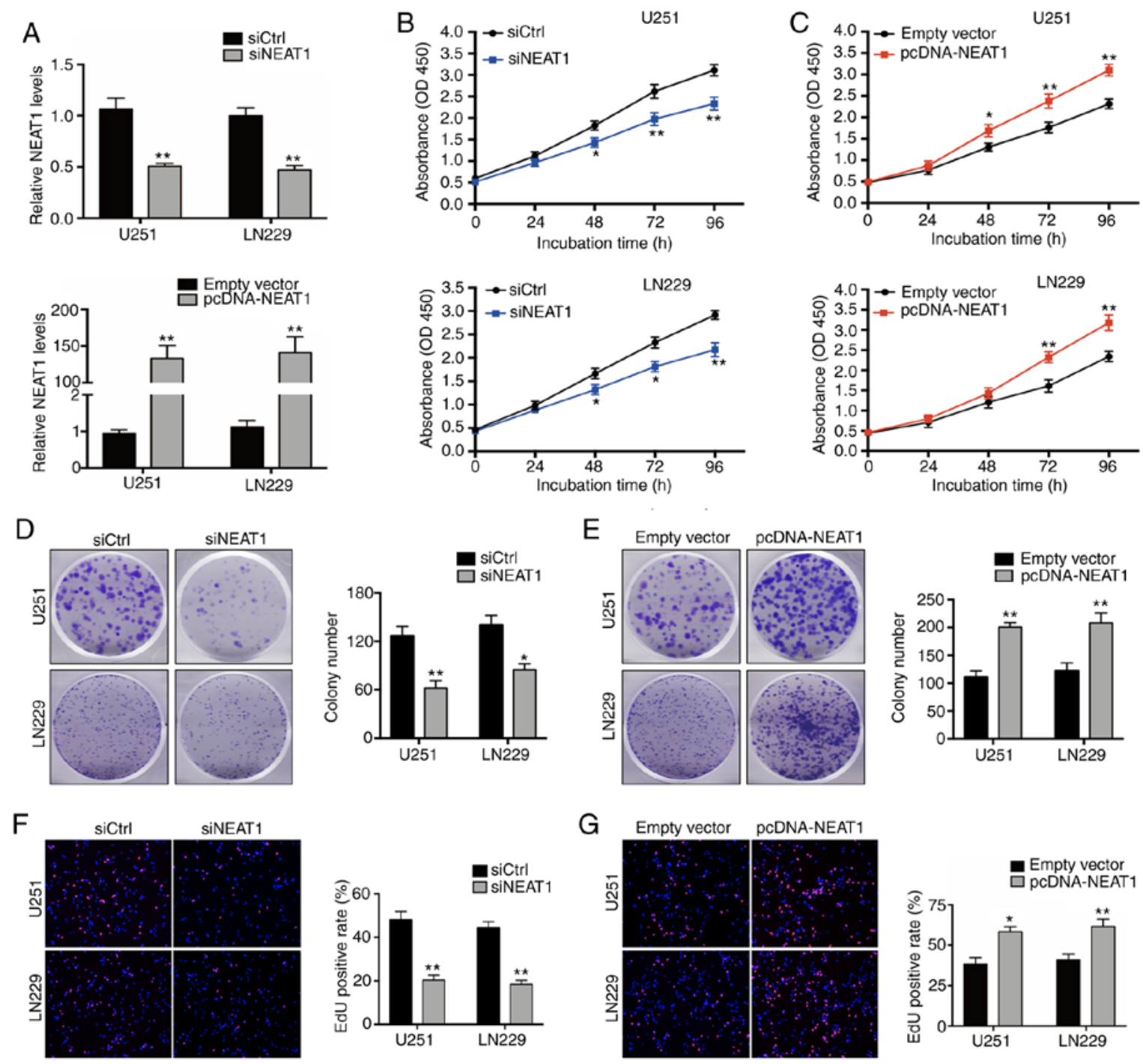

E
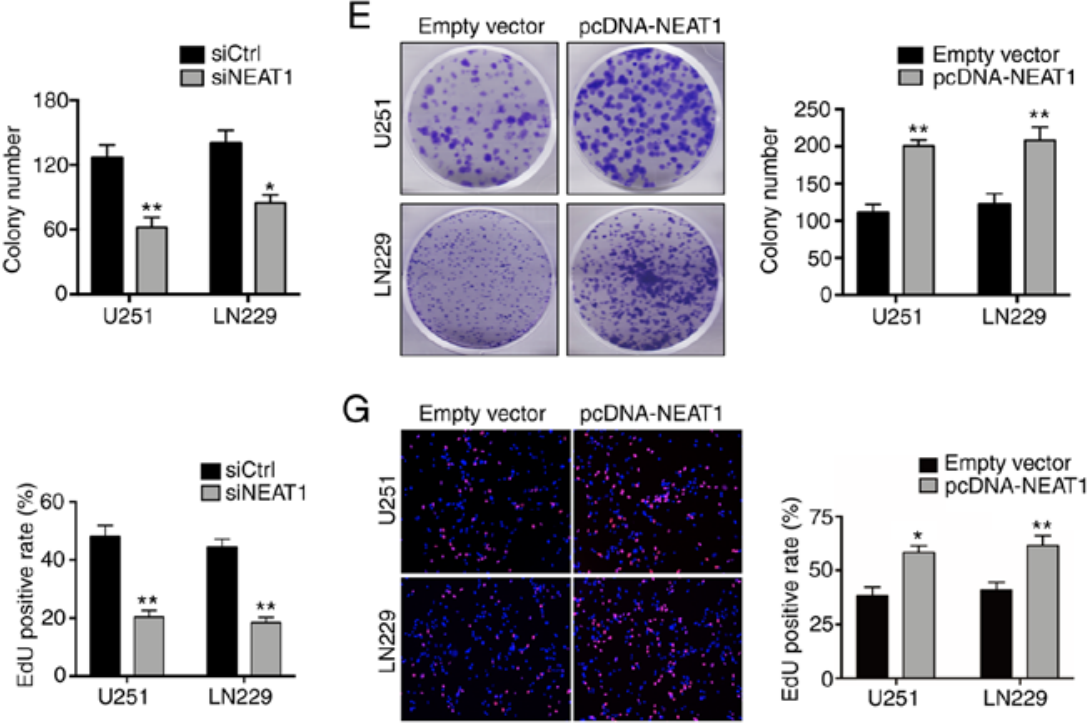

Figure 2. Effects of NEAT1 on glioma cells proliferation in vitro. (A) Reverse transcription-quantitative PCR analysis of NEAT1 expression in U251 and LN229 cells transfected with siCtrl, siNEAT1, pcDNA-NEAT1 or empty vector. (B) Cell Counting Kit-8 proliferation assay of U251 and LN229 cells transfected with siCtrl or siNEAT1. (C) A Cell Counting Kit-8 assay was used to analyze cell proliferation in U251 and LN229 cells transfected with empty vector or pcDNA-NEAT1. (D) Representative plate images (left) and quantitative analysis (right) of colony formation (magnification, $\mathrm{x} 40$ ) after 14 days of incubation of cells transfected with siCtrl or siNEAT1. (E) Representative plate images (left) and quantitative analysis (right) of colony formation (magnification, $\mathrm{x} 40$ ) after 14 days of incubation of cells transfected with empty vector or pcDNA-NEAT1. (F) Fluorescence microscopy images (left) and quantification (right) of EdU (magnification x200) staining of cells transfected with siCtrl or siNEAT1. (G) EdU (magnification x200) staining of cells transfected with empty vector or pcDNA-NEAT1. The experiments were performed in triplicate and data are presented as the mean $\pm \mathrm{SD}$. ${ }^{*} \mathrm{P}<0.05$ and ${ }^{* *} \mathrm{P}<0.01$ vs. siCtrl or empty vector. NEAT1, nuclear paraspeckle assembly transcript 1; EdU, ethynyldeoxyuridine; OD, optical density; pcDNA-NEAT1, NEAT1 overexpression vector; siRNA, small interfering RNA; siCtrl, control siRNA; siNEAT1, NEAT1-specific siRNA.

expressing shNC or shNEAT ( $\mathrm{n}=6$ for each group) were generated, and the cells were injected intracranially into nude mice. As shown in Fig. 3F and G, silencing of NEAT1 markedly inhibited the growth of intracranial tumors at all examined time points and also significantly increased the survival of mice. Furthermore, RT-qPCR revealed that the shNEAT1 transfection group had lower NEAT1 levels compared with the control group (Fig. $3 \mathrm{H}$ ). H\&E and IHC staining of tumors excised from experimental mice demonstrated the effect of NEAT1 knockdown on tumor growth. Tumors derived from shNEAT1-expressing U251 cells were markedly smaller and expressed much lower levels of Ki-67 compared with control tumors (Fig. 3I). These data indicated that silencing of NEAT1 inhibited glioma cell proliferation by inducing cell cycle arrest and promoting apoptosis, and demonstrated the carcinogenic activity of NEAT1 in glioma in vivo.

NEAT1 functions as a ceRNA by competitively binding to $m i R-324-5 p$. To determine whether the effects of NEAT1 in glioma might be mediated by sponging of $\geq 1$ miRNAs, the present study searched for potential candidate miRNAs with sequences complementary to NEAT1 using the online target prediction tools StarbaseV2.0 and lncRNASNP2. Based on this analysis, 13 miRNAs that were identified by both tools were selected (Fig. 4A), and their expression levels were analyzed in normal and glioma tissues using the TCGA and CGGA datasets. The results identified four miRNAs (miR-125a, miR-324-5p, miR-495-3p and miR-504) that were expressed at significantly lower levels in GBM tissues 
A

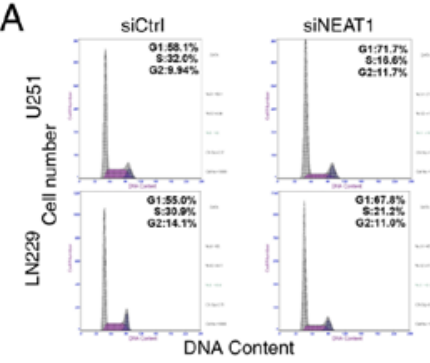

C siCtrI siNEAT1 1 Annexin V-FITC/PI (A) Annexin V-FITC/PI

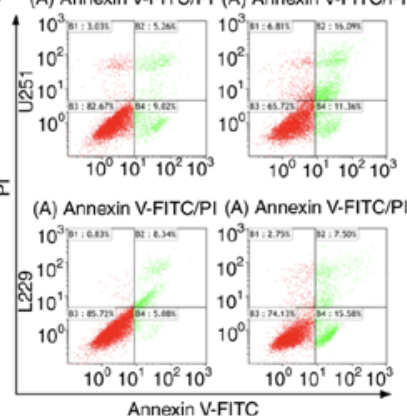

口G2/M口S Go/G1
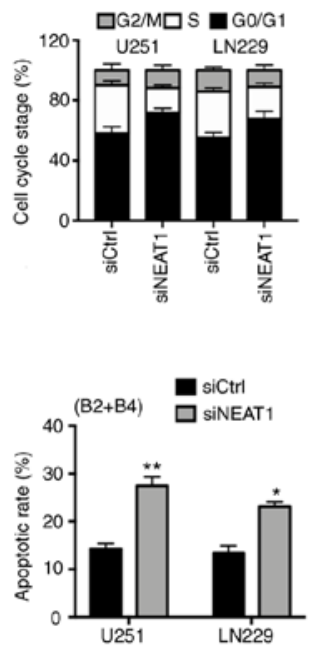

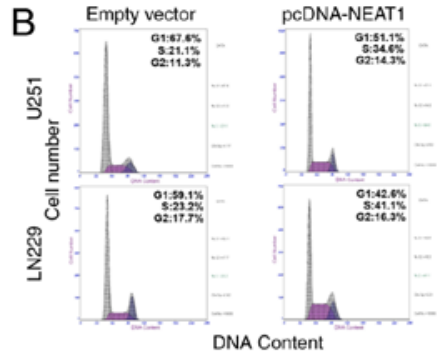

Empty vector pcDNA-NEAT1

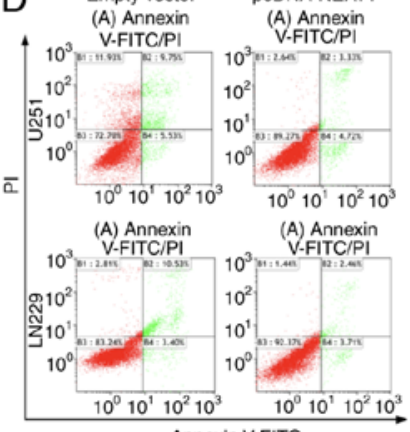

Annexin V-FITC
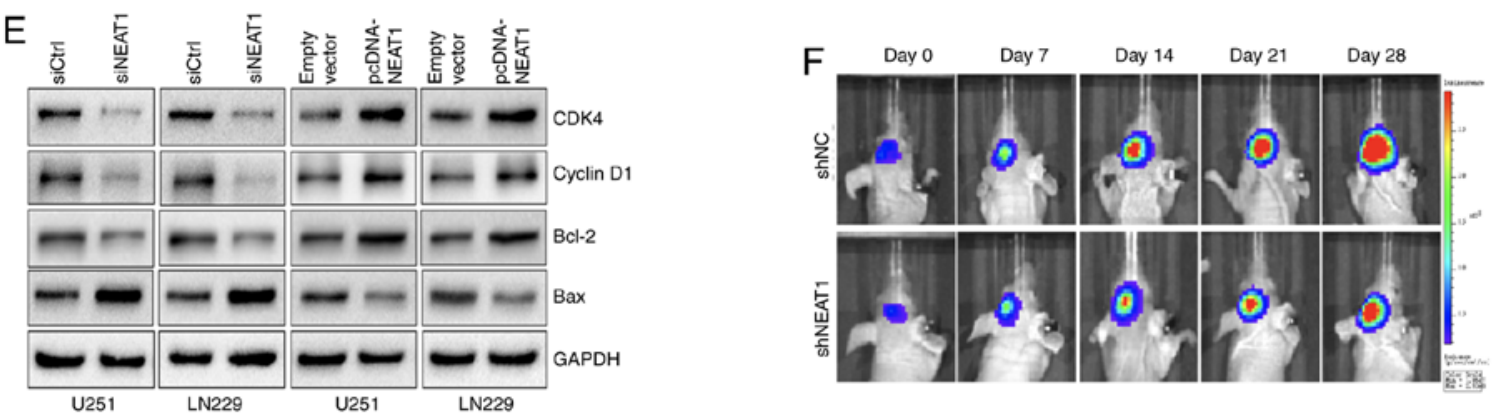

G

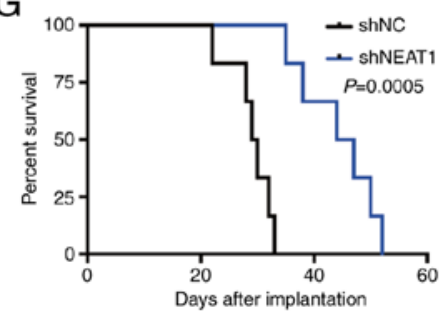

$\mathrm{H}$

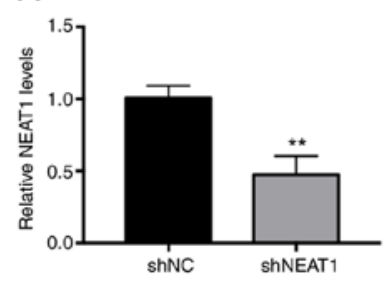

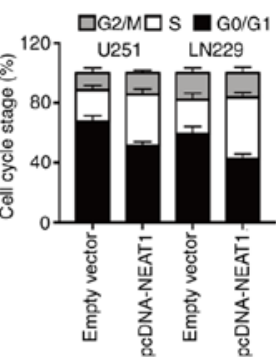

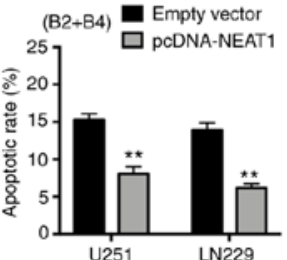

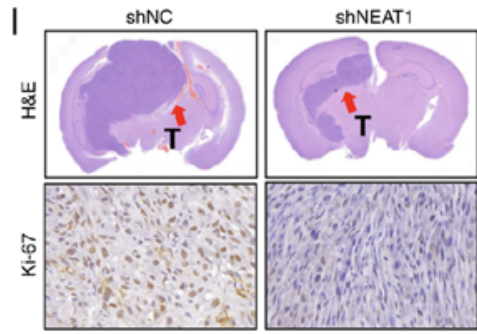

Figure 3. Long non-coding RNA NEAT1 involvement in glioma cell apoptosis and cell cycle progression in vitro and in vivo. (A) Flow cytometry histograms (left) and quantification (right) of the cell cycle distribution of U251 and LN229 cells transfected with siCtrl or siNEAT1. (B) Flow cytometry assays were performed to analysis the cell cycle distribution of U251 and LN229 cells transfected with pcDNA-NEAT1 or empty vector. (C) Flow cytometry dot plots (left) and quantification (right) of the apoptotic rates of U251 and LN229 cells transfected with siCtrl or siNEAT1. Quadrants B2 and B4 contained terminal and early apoptotic cells, respectively. (D) Flow cytometry cell apoptosis assays were performed to measure the apoptotic rates of U251 and LN229 cells transfected with pcDNA-NEAT1 or empty vector. Quadrants B2 and B4 contained terminal and early apoptotic cells, respectively. (E) Western blot analysis of the expression levels of cell cycle proteins (CDK4 and cyclin D1) and apoptosis-related proteins (Bcl-2 and Bax) in U251 and LN229 cells transfected as described for (A and B) GAPDH was used as the loading control. (F) Representative in vivo images of nude mice injected intracranially with U251 cells transfected with shNC or shNEAT1 ( $\mathrm{n}=6$ for each group). Mice were injected with D-luciferin and imaged with the IVIS imaging system. (G) Survival of mice treated as described for $(\mathrm{F}) \mathrm{A}$ log-rank test was used to assess the statistical significance of the differences. (H) Reverse transcription-quantitative PCR was performed to detect the expression levels of NEAT1 in implanted tumor tissues. (I) Representative images of H\&E staining (upper panels; magnification, $\mathrm{x} 100$ ) and immunohistochemical staining of Ki-67 (lower panels; magnification, $\mathrm{x} 400$ ) of tumors excised from mice treated with shNEAT1. Arrows indicate the location of the tumor. The experiments were performed in triplicate and data are presented as the mean $\pm \mathrm{SD}$. ${ }^{*} \mathrm{P}<0.05$ and ${ }^{* *} \mathrm{P}<0.01 \mathrm{vs}$. siCtrl or empty vector. NEAT1, nuclear paraspeckle assembly transcript 1; pcDNA-NEAT1, NEAT1 overexpression vector; shNC, control shRNA; shNEAT1, NEAT1-specific shRNA; shRNA, short hairpin RNA; siCtrl, control siRNA; siNEAT1, NEAT1-specific siRNA; siRNA, small interfering RNA; T, tumor.

compared with normal brain tissues (Figs. 4B and S1A), and in HGG compared with LGG (Fig. 4C).

The ability of miR-125a, miR-324-5p, miR-495-3p and miR-504 to interact directly with NEAT1 was analyzed using luciferase reporter assays. Co-transfection of cells with a NEAT1-driven luciferase expression vector and miR-125a or
miR-324-5p mimics significantly inhibited luciferase activity compared with that of cells co-transfected with controls (Fig. 4D), indicating that these two miRNAs bound to the NEAT1 sequence. Of the two inhibitory miRNAs, miR-324-5p had the greatest inhibitory activity and was therefore selected for further analysis. To verify binding between NEAT1 and 

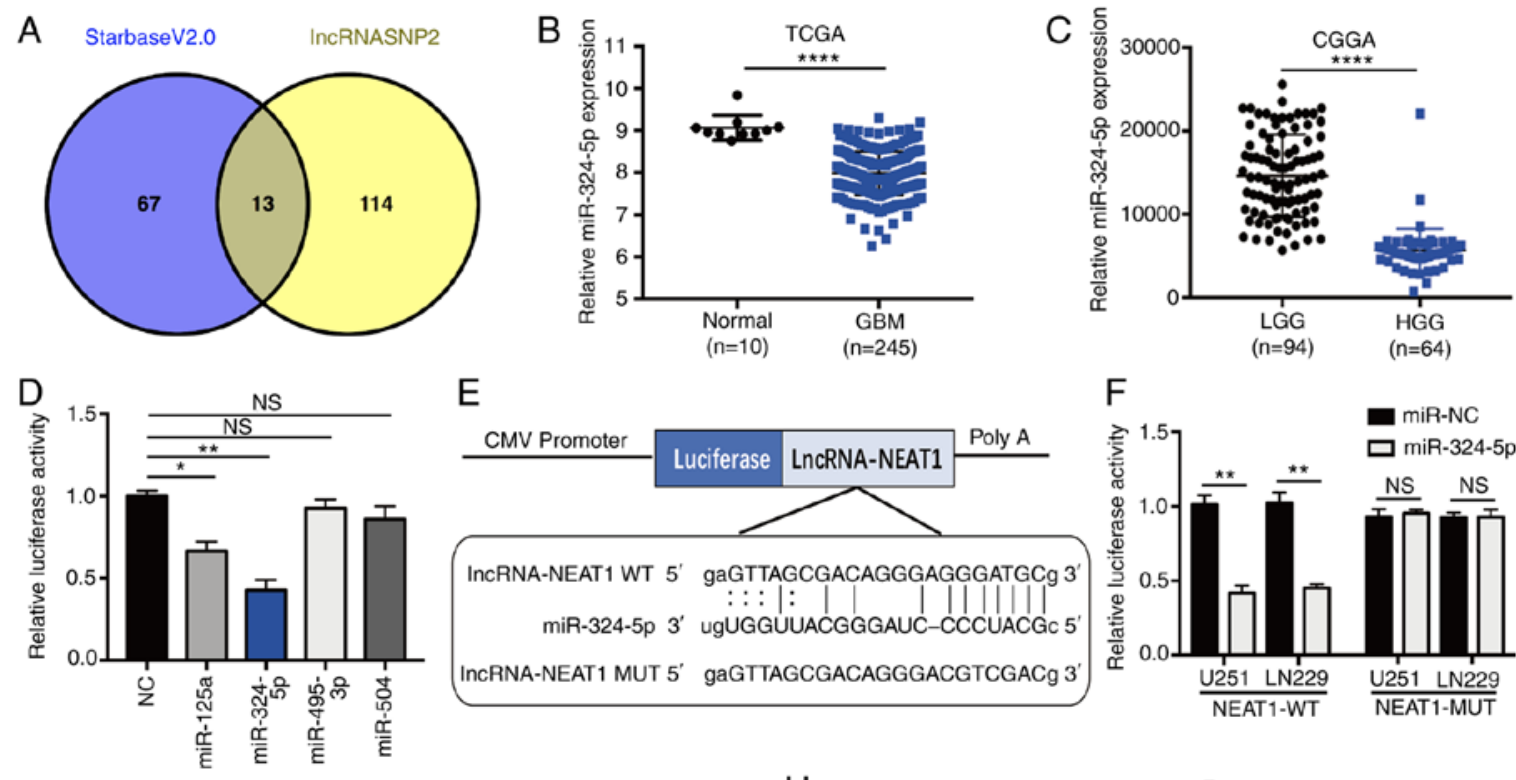

E
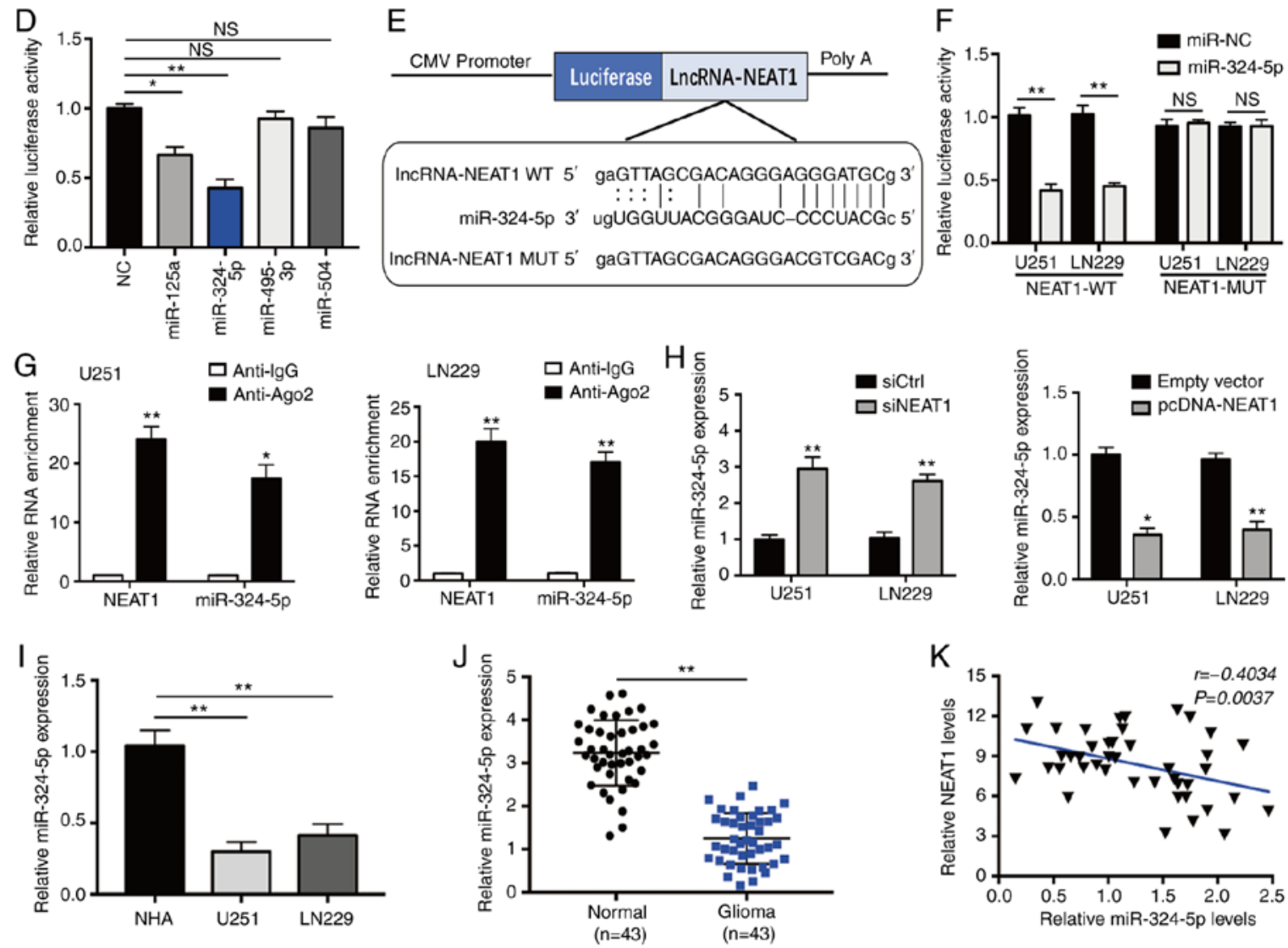

Figure 4. Direct interaction between lncRNA NEAT1 and miR-324-5p. (A) Venn diagram showing an overlap of potential NEAT1 targets predicted by Starbase V2.0 and lncRNASNP2. (B and C) Relative expression levels of miR-324-5p in glioma tissues compared with normal tissues. Data were obtained from (B) TCGA and (C) CGGA databases. (D) Luciferase activity in 293T cells co-transfected with the luciferase reporter gene plasmids containing NEAT1 sequence and four different miR-encoding plasmids. (E) Schematic showing the WT sequence of NEAT1, the location of the miR-324-5p-binding site predicted by lncRNASNP2, and the MUT sequence of NEAT1 used for the luciferase reporter assays. (F) Luciferase activity of U251 and LN229 cells co-transfected with NC or miR-324-5p mimics and a luciferase reporter plasmid driven by WT- or MUT-NEAT1. (G) RNA immunoprecipitation assays of U251 and LN229 cells immunoprecipitated with IgG or anti-Ago2 antibodies, followed by RT-qPCR of the immmunoprecipitates for NEAT1 and miR-324-5p sequences. (H) RT-qPCR of miR-324-5p expression in U251 and LN229 cells transfected with siCtrl, siNEAT1, pcDNA-NEAT1 or empty vector. RT-qPCR analysis of miR-324-5p expression in (I) NHAs or U251 and LN229 cells, or (J) in glioma and matched normal tissues. (K) Spearman's rank correlation analysis of the association between NEAT1 and miR-324-5p expression in 43 glioma specimens. The experiments were performed in triplicate and data are presented as the mean \pm SD. ${ }^{*} \mathrm{P}<0.05,{ }^{* *} \mathrm{P}<0.01$ and ${ }^{* * * * *} \mathrm{P}<0.0001$ vs. anti-IgG, siCtrl or empty vector. Ago2, argonaute RISC catalytic component 2; CGGA, Chinese Glioma Genome Atlas; GBM, glioblastoma; HGG, high-grade glioma; LGG, low-grade glioma; lncRNA, long non-coding RNA; miR, microRNA; MUT, mutant; NC, negative control; NEAT1, nuclear paraspeckle assembly transcript 1; NHAs, normal human astrocytes; NS, not significant; pcDNA-NEAT1, NEAT1 overexpression vector; RT-qPCR, reverse transcription quantitative PCR; siCtrl, control siRNA; siNEAT1, NEAT1-specific siRNA; siRNA, small interfering RNA; TCGA, The Cancer Genome Atlas; WT, wild-type.

miR-324-5p, the control or miR-324-5p mimics were co-transfected along with luciferase vectors driven by the WT-NEAT1 sequence or a mutated sequence (MUT-NEAT1) carrying mismatched residues in the predicted miR-324-5p binding site (Fig. 4E). As shown in Fig. 4F, the luciferase activity of U251 and LN229 cells co-transfected with WT-NEAT1 and miR-324-5p was significantly reduced compared with that of cells transfected with miR-NC, but there was no significant change in the MUT-NEAT1 group, which demonstrated a direct association between NEAT1 and miR-324-5p. These results were substantiated by the results of the RIP experiments in U251 and LN229 cells. The RIP analysis revealed that NEAT1 was highly enriched in Ago2 immmunoprecipitates from cells transfected with the miR-324-5p mimics compared with the anti-IgG group (Fig. 4G).

The assessment of the functional association between NEAT1 and miR-324-5p by RT-qPCR, and it was revealed that knockdown of NEAT1 was associated with the upregulation 

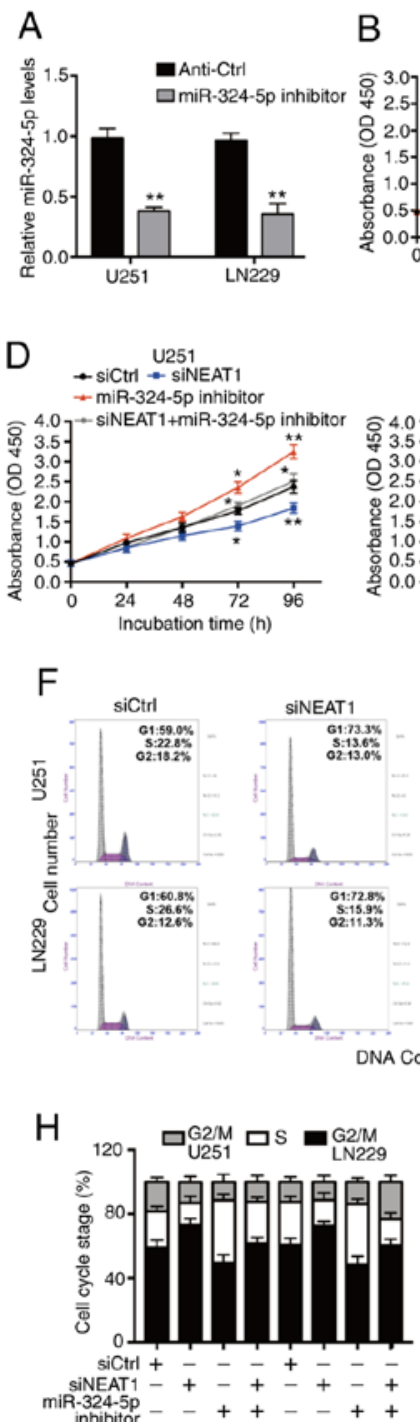

$\mathrm{B}$
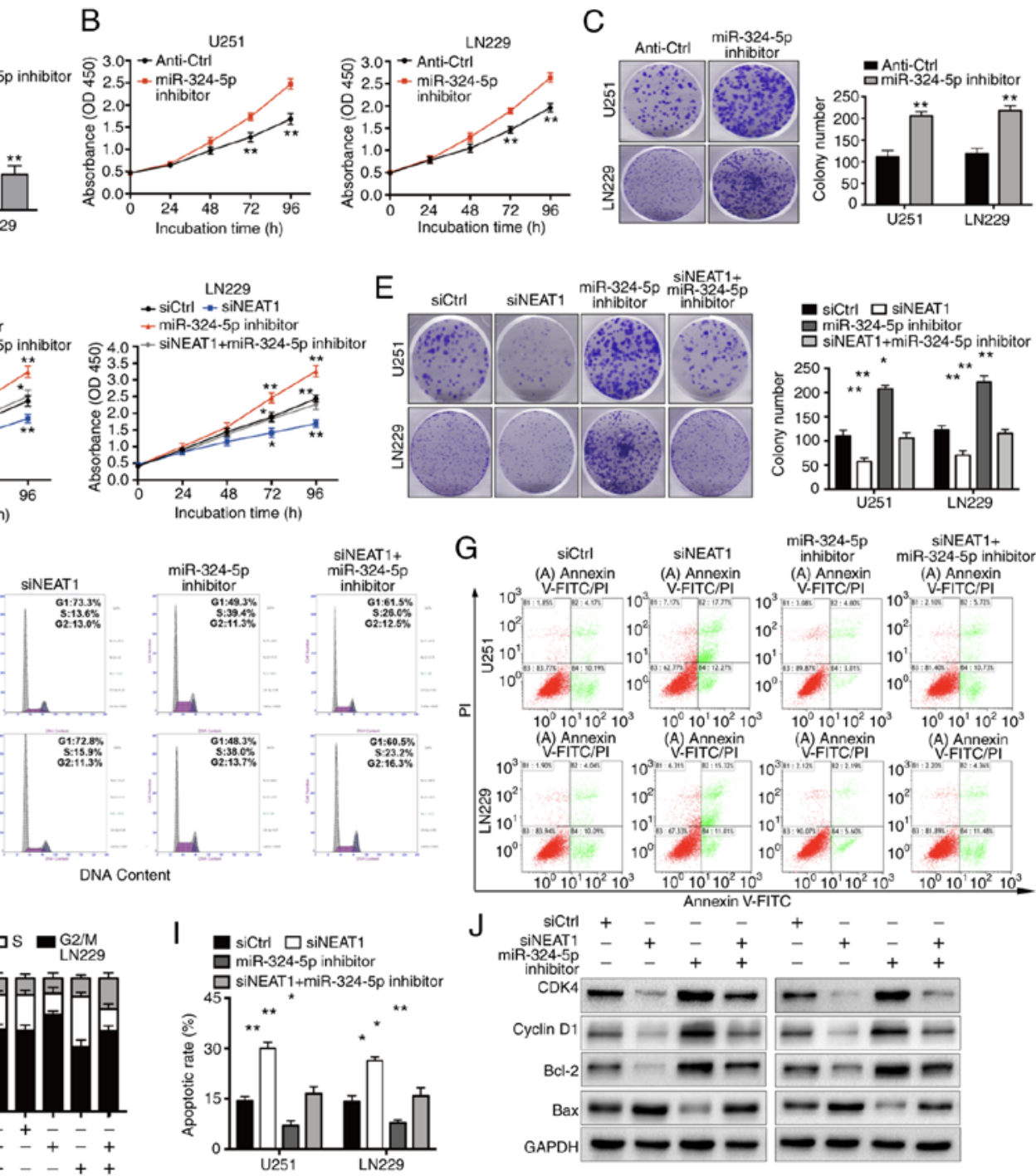

Figure 5. miR-324-5p involvement in glioma cell proliferation, cell cycle progression and apoptosis in vitro. (A) Reverse transcription-quantitative PCR analysis of miR-324 expression, (B) CCK-8 cell proliferation assay and (C) colony formation assay (magnification, $\mathrm{x} 40$ ) of U251 and LN229 cells transfected with an miR-324-5p inhibitor or Anti-Ctrl. (D) CCK-8 cell proliferation assay, (E) colony formation assay (magnification, $\mathrm{x} 40$ ) and (F and H) cell cycle distribution analysis of U251 and LN229 cells transfected with siCtrl or siNEAT1 with or without an miR-324-5p inhibitor. (G and I) FACS analysis of the apoptotic rate of U251 and LN229 cells transfected with siCtrl or siNEAT1 with or without an miR-324-5p inhibitor. (J) Western blotting of the indicated cell cycle and apoptosis proteins in LN229 and U251 cells transfected as described for (F-I) GAPDH was used as the loading control. The experiments were performed in triplicate and data are presented as the mean \pm SD. ${ }^{*} \mathrm{P}<0.05$ and ${ }^{* *} \mathrm{P}<0.01$ vs. Anti-Ctrl, siCtrl or as indicated. Anti-Ctrl, control sequence; CCK-8, Cell Counting Kit-8; FACS, fluorescence activated cell sorting; miR, microRNA; NEAT1, nuclear paraspeckle assembly transcript 1; OD, optical density; siCtrl, control siRNA; siNEAT1, NEAT1-specific siRNA; siRNA, small interfering RNA.

of miR-324-5p in U251 and LN229 cells. Furthermore, overexpression of NEAT1 was associated with the opposite results (Fig. 4H). The downregulation or upregulation of miR-324-5p had no significant effect on NEAT1 expression (Fig. S1B). These results demonstrated that NEAT1 may directly bind to and modulate the expression levels of miR-324-5p in glioma cells. The expression levels of miR-324-5p were detected in NHA, U251 and LN229 cells by RT-qPCR, and the results revealed that, compared those in with NHA cells, the expression levels of miR-324-5p in U251 and LN229 were significantly reduced (Fig. 4I). Furthermore, miR-324-5p expression was significantly lower in human glioma tissues compared with in normal tissues (Fig. 4J), and Spearman's correlation analysis demonstrated a significant negative correlation between the expression levels of NEAT1 and miR-324-5p in glioma tissues (Fig. 4K). In combination, these data demonstrated that NEAT1 functions as a ceRNA to modulate miR-324-5p levels in glioma.

miR-324-5p inhibition reverses the suppressive effects of knockdown of NEAT1 on glioma cells. Having established an association between NEAT1 and miR-324-5p, it was next examined whether miR-324-5p mediates the NEAT1-induced changes in glioma cell biology. For these experiments, U251 and LN229 cells were transfected with a miR-324-5p inhibitor (Fig. 5A) with or without concomitant NEAT1 knockdown. Compared with those of control glioma cells, transfection of the miR-324-5p inhibitor alone markedly increased proliferation and colony formation (Fig. 5B and C). In addition, the experiments demonstrated that overexpression of miR-324-5p 
A

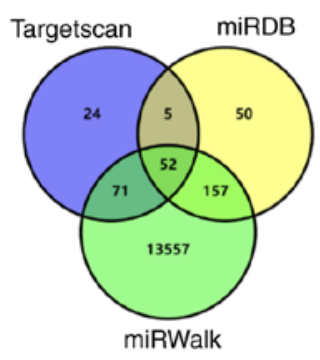

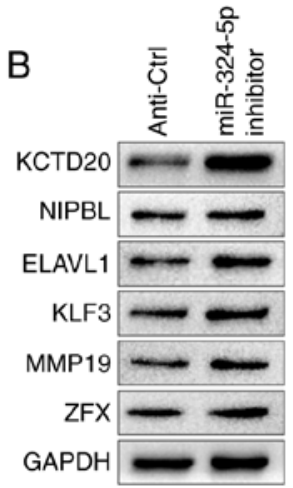

U251

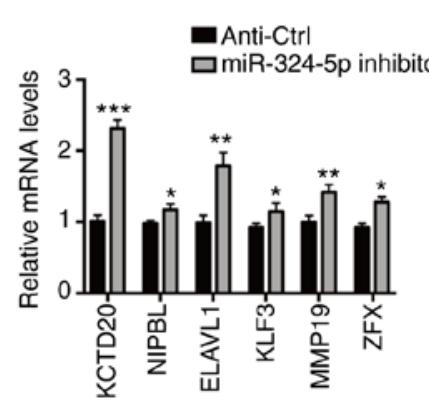

C

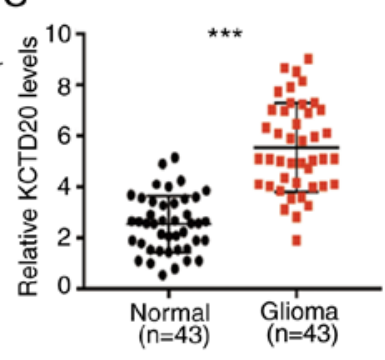

$\mathrm{E}$

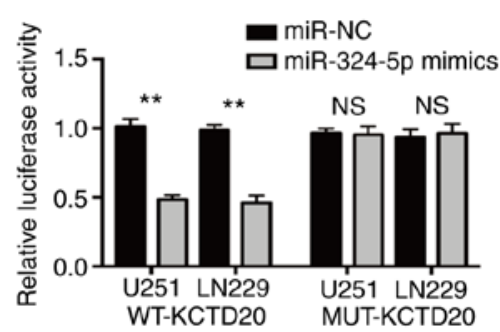

G
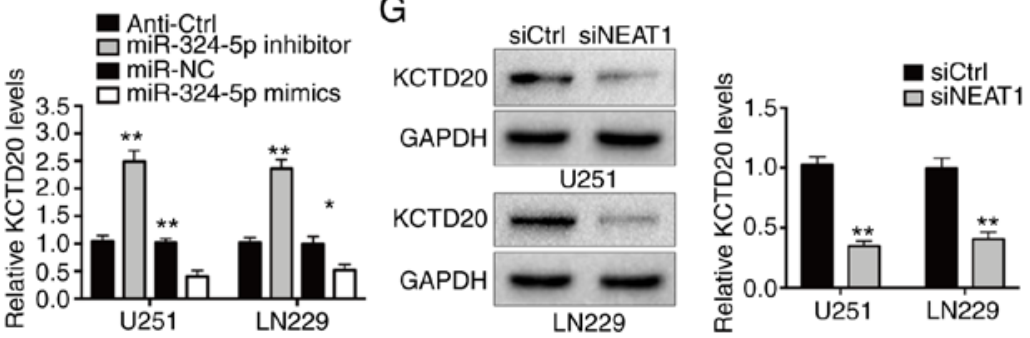

।
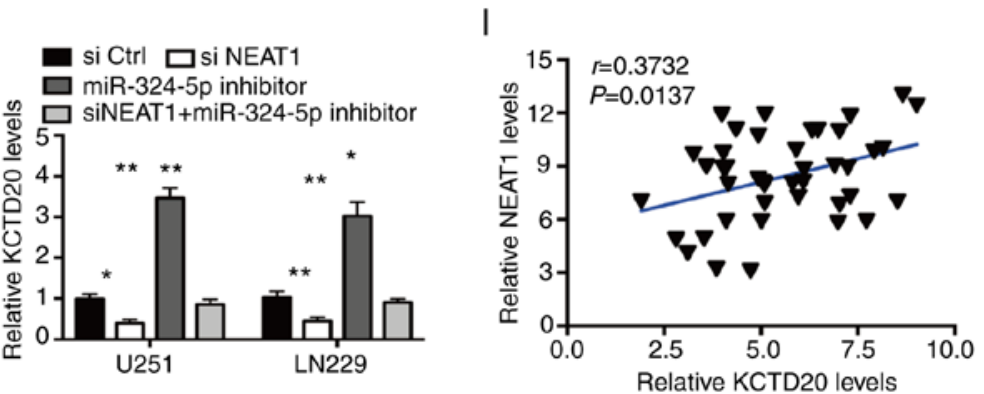

Figure 6. Direct targeting of the 3'-UTR of KCTD20 by miR-324-5p and inhibition of its effects by silencing of NEAT1. (A) Venn diagram showing an overlap among potential miR-324-5p target genes predicted by Targetscan, miRBD and miRWalk. (B) Western blotting and RT-qPCR analysis of the expression levels of six putative miR-324-5p target genes in U251 cells transfected with Anti-Ctrl or miR-324-5p inhibitor. (C) RT-qPCR analysis of KCTD20 expression in glioma and adjacent non-tumor tissues. (D) Schematic showing the WT-KCTD20 3'-UTR sequence, the location of the miR-324-5p binding site predicted by Targetscan and the MUT-KCTD20 3'-UTR sequence used for the luciferase reporter assay. (E) Luciferase activity in U251 and LN229 cells co-transfected with a luciferase plasmid driven by the WT- or MUT-KCTD20 3'-UTR and either control (miR-NC) or miR-324-5p mimics. (F) Western blotting and RT-qPCR of KCTD20 expression in U251 and LN229 cells transfected with Anti-Ctrl, miR-324-5p inhibitor, miR-NC or miR-324-5p mimics. (G) Western blotting and RT-qPCR analysis of KCTD20 expression in U251 and LN229 cells transfected with siCtrl or siNEAT1. (H) Western blotting and RT-qPCR analysis of KCTD20 expression in U251 and LN229 cells transfected with siCtrl or siNEAT1 with or without an miR-324-5p inhibitor. (I) Spearman's rank correlation analysis of the association between NEAT1 and KCTD20 mRNA expression in 43 glioma tissues. The experiments were performed in triplicate and data are presented as the mean $\pm \mathrm{SD}$. ${ }^{*} \mathrm{P}<0.05,{ }^{* * *} \mathrm{P}<0.01$ and ${ }^{* * *} \mathrm{P}<0.001$ vs. Anti-Ctrl, miR-NC, siCtrl or as indicated. Anti-Ctrl, control sequence; ELAVL1, ELAV like RNA binding protein 1; KCTD20, potassium channel tetramerization protein domain containing 20; KLF3, Kruppel like factor 3; miR, microRNA; MUT, mutant; NC, negative control; NEAT1, nuclear paraspeckle assembly transcript 1; NIPBL, NIPBL cohesin loading factor; RT-qPCR, reverse transcription quantitative polymerase chain reaction; siCtrl, control siRNA; siNEAT1, NEAT1-specific siRNA; siRNA, small interfering RNA; 3'UTR, 3' untranslated region; WT, wild-type; ZFX, zinc finger protein X-linked.

significantly inhibited the progression of glioma in vitro. miR-324-5p mimics were transfected into U251 and LN229 cells (Fig. S1C). Colony formation and EdU assays revealed that overexpression of miR-324-5p significantly inhibited the cell proliferation rate (Fig. S1D and E). Additionally, the cell cycle was blocked at the $\mathrm{G}_{0} / \mathrm{G}_{1}$ phase (Fig. S1F). Additionally, upregulation of miR-324-5p resulted in an increase in the number of apoptotic cells (Fig. S1G). Western blotting was 
A

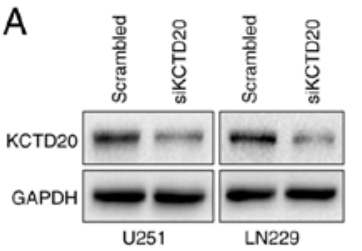

C

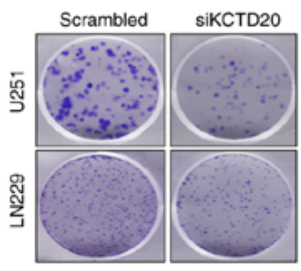

E

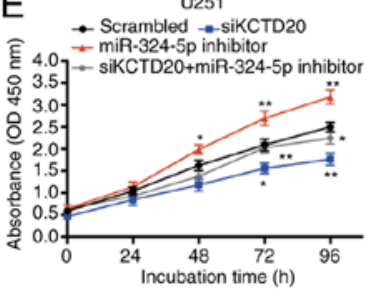

G

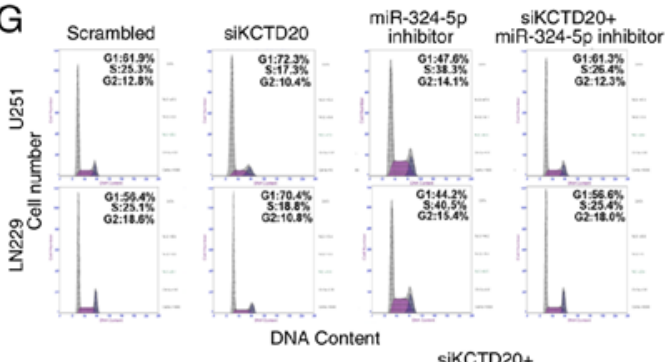

B
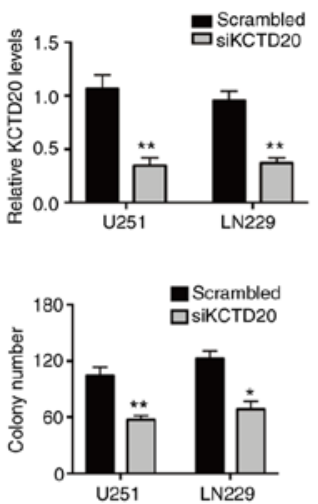

B.

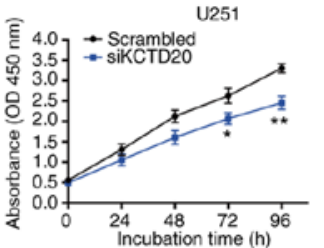

D

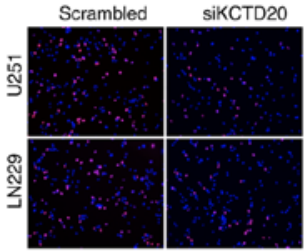

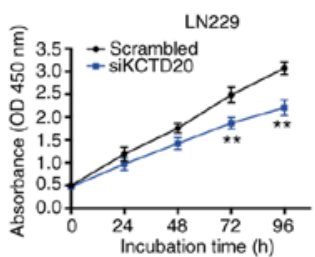

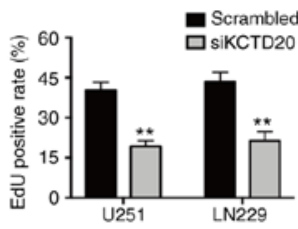

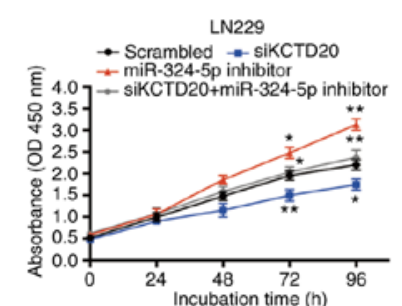

F

F Scrambled sikCTD20 miR-324-5p $\begin{gathered}\text { sikCTD20+ } \\ \text { miR-324-5p } \\ \text { inhibitor }\end{gathered}$

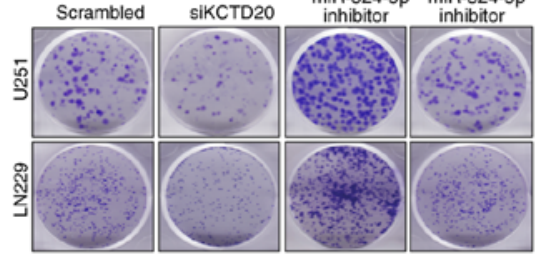

$\mathrm{H}$

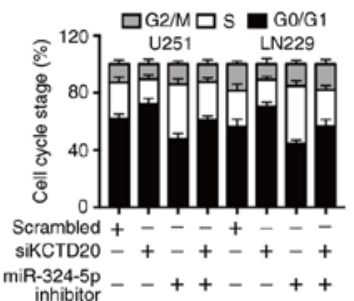

Scrambled $\square$ sikCTD20 miR-324-5p inhibitor siKCTD20+miR-324-5p inhibitor
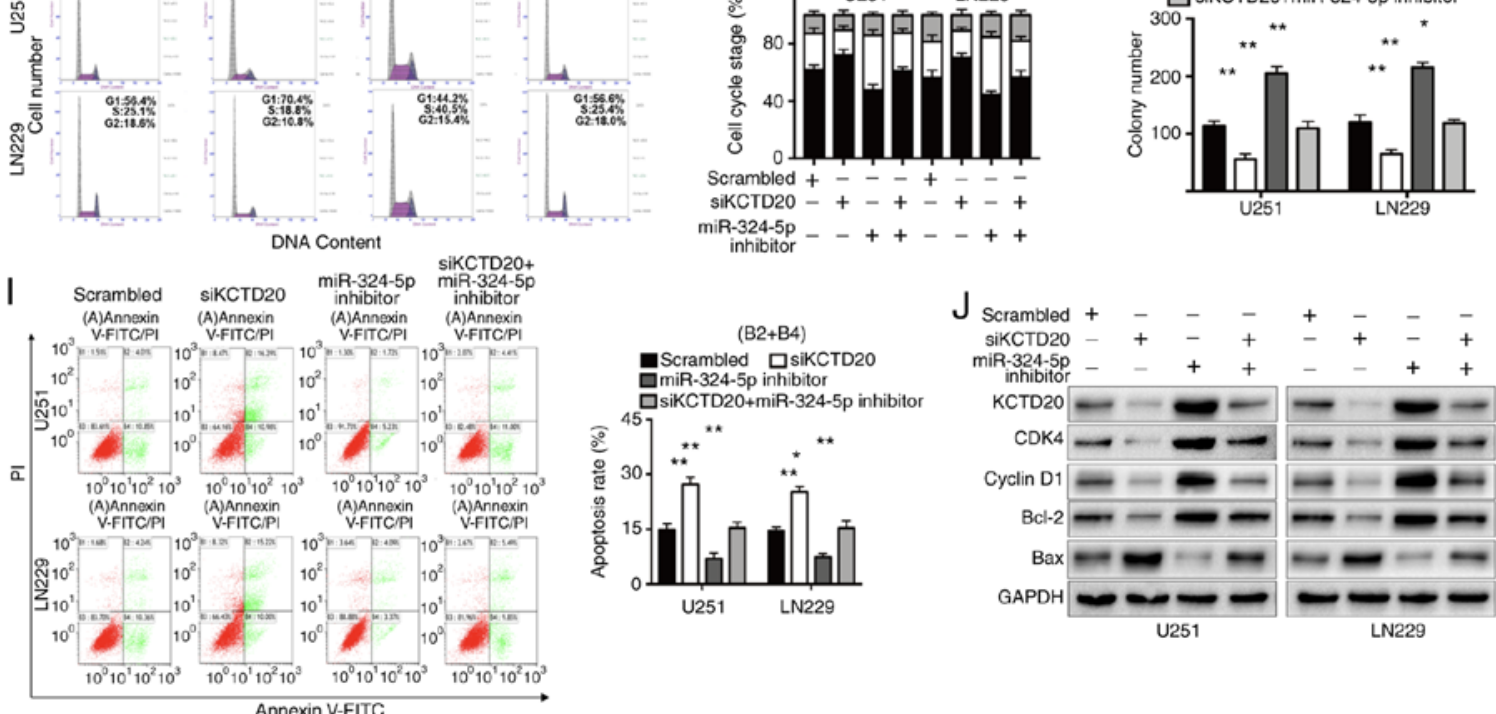

Figure 7. Inhibition of glioma cell proliferation and induction of cell cycle arrest and apoptosis in vitro by knockdown of KCTD20. (A) Western blotting and Reverse transcription-quantitative PCR analysis of KCTD20 expression in U251 and LN229 cells transfected with a scrambled control sequence or siKCTD20. (B) Cell Counting Kit-8 proliferation assay, (C) colony formation assay (magnification, x40) and (D) EdU (magnification x200) staining of U251 and LN229 cells transfected with a scrambled sequence or siKCTD20. (E-H) Rescue of siKCTD20-induced inhibition of (E) cell proliferation, (F and H) cell colony formation (magnification, $\mathrm{x} 40$ ), (G) cell cycle progression and (I) apoptosis in U251 and LN229 cells by co-transfection with an miR-324-5p inhibitor. (J) Western blot analysis of the expression levels of KCTD20, cell cycle proteins (CDK4 and cyclin D1), apoptosis-related proteins (Bcl-2 and Bax) and GAPDH in $\mathrm{U} 251$ and LN229 cells transfected as described for $(\mathrm{G}$ and $\mathrm{H})$ The experiments were performed in triplicate and data are presented as the mean \pm SD. ${ }^{*} \mathrm{P}<0.05$ and ${ }^{* *} \mathrm{P}<0.01$ vs. scrambled or as indicated. EdU, ethynyldeoxyuridine; KCTD20, potassium channel tetramerization protein domain containing 20; miR, microRNA; OD, optical density; siKCTD20, KCTD20-targeting small interfering RNA.

used to analyze the expression levels of cycle-related proteins and apoptosis-related proteins after cells were transfected with miR-324-5p mimics (Fig. S1H). As shown in Fig. 5E-J, glioma cells were co-transfected with siNEAT1 and the miR-324-5p inhibitor or siCtrl. These experiments demonstrated that miR-324-5p inhibition partially reversed the effects of transfection with siNEAT1 on U251 and LN229 cell proliferation and colony formation (Fig. 5D and E), as well as cell cycle arrest and apoptosis (Fig. 5F-I), and had a corresponding rescue effect on the expression levels of cell cycle and apoptosis-related proteins (Fig. 5J). Collectively, these data suggested that a potential interaction between NEAT1 and miR-324-5p may be involved in the development of glioma.

NEAT1 positively regulates KCTD20 expression via competitive inhibition of miR-324-5p. The present study next 
sought to identify the target mRNAs of miR-324-5p through which NEAT1 and miR-324-5p regulate glioma cell proliferation. Using the online informatics tools TargetScan, miRDB and miRWalk, which predict miR-324-5p target mRNAs based on sequence complementarity, 52 shared target genes with potential binding sites for miR-324-5p were identified from the three online informatics tools (Fig. 6A). Among the 52 genes, six were expressed at significantly higher levels in glioma tissues compared with normal tissues in the dataset from TCGA (Fig. S2A and B). The mRNA and protein expression levels of the six genes were analyzed in U251 cells transfected with a control sequence or the miR-324-5p inhibitor. This analysis revealed that miR-324-5p suppression significantly increased the expression levels of all six genes. However, the most striking effect was that on KCTD20 expression (Fig. 6B). Notably, KCTD20 expression was consistently upregulated in glioma tissues compared with normal brain tissues in the clinical specimens, as well as the TCGA, CGGA and GSE16011 datasets (Figs. 6C and S2B). Similarly, IHC staining showed that the abundance of KCTD20 protein in human glioma samples was increased compared with that in normal brain tissue (Fig. S2C). Therefore, KCTD20 was selected for further analysis of the NEAT1-miR-324-5p-mRNA regulatory relationship in glioma cells.

To verify that KCTD20 mRNA was directly regulated by miR-324-5p in glioma cells, a luciferase reporter assay was performed in cells expressing plasmids with the WT 3'-UTR of KCTD20 or MUT 3'-UTR carrying mutations in the putative binding site for miR-324-5p (Fig. 6D). Co-transfection with miR-324-5p mimics significantly repressed luciferase activity in U251 and LN229 cells expressing the WT, but not the MUT, KCTD20 3'-UTR (Fig. 6E). Furthermore, KCTD20 protein and mRNA expression was increased following the transfection of glioma cells with the miR-324-5p inhibitor and suppressed by their transfection with miR-324-5p mimics (Fig. 6F). These data demonstrated that miR-324-5p functionally regulated KCTD20 expression in glioma cells and suggested that KCTD20 expression may therefore be regulated by changes in NEAT1 levels. To test this, KCTD20 expression was examined by western blotting and RT-qPCR in cells transfected with siNEAT1 or a control sequence. Notably, KCTD20 expression was markedly reduced following NEAT1 knockdown (Fig. 6G). However, co-silencing of miR-324-5p abolished the inhibitory effects of siNEAT1 on KCTD20 mRNA and protein expression (Fig. 6H). IHC staining revealed reduced KCTD20 expression in tumor sections from mice injected with shNEAT1-expressing glioma cells compared with in mice injected with control cells (Fig. S2D). Finally, a significant positive correlation was identified between the mRNA expression levels of NEAT1 and KCTD20 in the glioma specimens (Fig. 6I). Since NEAT1 could sponge miR-324-5p, the present study next determined whether NEAT1 could regulate KCTD20 expression by binding to the same site in miR-204-5p. Luciferase reporter assays demonstrated that miR-324-5p could bind to NEAT1 and the 3' UTR of KCTD20. To determine whether miR-324-5p served a role in the relationship between NEAT1 and KCTD20, cells were co-transfected with siNEAT1 and the miR-324-5p inhibitor. Knockdown of NEAT1 also significantly reduced KCTD20 mRNA and protein expression in U251 and LN229 cells
(Fig. 6G). Additionally, the downregulation of KCTD20 protein expression induced by siNEAT1 was effectively reversed by the miR-324-5p inhibitor. The experimental results also showed that changes in the expression of NEAT1 could affect the expression levels of miR-324-5p; however, changes in the expression levels of miR-324-5p had no effect on NEAT1 (Fig. S1B). The expression levels of NEAT1 and KCTD20 in clinical tissues were positively associated (Fig. 6I), consistent with the existence of a NEAT1-miR-324-5p-KCTD20 regulatory axis. In combination, these data suggested that NEAT1 regulated the expression levels of KCTD20 in glioma cells by post-transcriptional modulation of miR-324-5p.

KCTD20 mediates the effects of NEAT1 on glioma cell proliferation in vitro and in vivo. Subsequently, it was determined whether the functional effects of NEAT1 and miR-324-5p on glioma cell biology were mediated through their control of KCTD20 expression. Transfection of U251 and LN229 cells with siKCTD20 effectively decreased KCTD20 mRNA and protein expression compared with that in control cells (Fig. 7A). Notably, the silencing of KCTD20 significantly inhibited U251 and LN229 proliferation, based on the results of CCK-8, colony formation and EdU incorporation assays (Fig. 7B-D), Furthermore, the results demonstrated that more cells were in the $G_{0} / G_{1}$ phase and fewer cells were in $S$ phase of the cell cycle after cells were transfected with siKCTD20 (Fig. S3A), Depletion of KCTD20 significantly increased the efficiency of apoptosis (Fig. S3B). Similarly, the protein expression levels of cyclin D1, CDK4 and Bcl-2 were downregulated, while the expression levels of Bax protein were upregulated (Fig. S3C). These results were consistent with the observed effects of knockdown of NEAT1 or overexpression of miR-324-5p on glioma cell functions.

The findings were verified by examining the effects of KCTD20 knockdown or concomitant KCTD20 and miR-324-5p knockdown on glioma cell biology. First, U251 cells stably expressing luciferase and either shKCTD20 or control shNC ( $\mathrm{n}=6$ for each group) were intracerebrally injected into nude mice, and the mice were followed up by imaging examinations for up to 28 days. Consistent with the results of the in vitro assays, silencing of KCTD20 markedly inhibited glioma growth and significantly improved the survival time of tumor-bearing mice (Fig. S3D and E). As presented in Fig. S3F, immunoblot analysis indicated that KCTD20 expression was downregulated in shKCTD20-inoculated tumor tissues. In addition, transfection of miR-324-5p inhibitor in addition to knockdown of KCTD20 in glioma cell lines partially reversed the effects of KCTD20 knockdown (Figs. 7E, F and $\mathrm{H}$ and $\mathrm{S} 3 \mathrm{G}$ and $\mathrm{H}$ ), demonstrating the functional relationship between miR-324-5p and KCTD20. Furthermore, the concomitant knockdown of miR-324-5p and KCTD20 in U251 and LN229 cells abrogated the effects of knockdown of KCTD20 on cell cycle arrest (Fig. 7G), apoptosis (Fig. 7I), and CDK4, cyclin D1, Bcl-2 and Bax expression (Fig. 7J). Finally, a statistically significant inverse correlation was detected between the expression levels of miR-324-5p and KCTD20 in the clinical glioma tissues $(\mathrm{r}=-0.4582 ; \mathrm{P}=0.002 ; \mathrm{Fig}$. S3I). In combination, the data presented in the current study provided substantial evidence that NEAT1 is oncogenic in glioma cells and exerts 
its effects through the modulation of miR-324-5p-regulated KCTD20 expression.

\section{Discussion}

In the last several decades, intense efforts have been made to identify lncRNAs and to elucidate their roles in the development and progression of several human diseases, including cancer (41-43). The roles of lncRNAs as important regulatory factors in cellular processes through their effects on gene expression via epigenetic, transcriptional and post-transcriptional mechanisms (44). Although numerous lncRNAs have been annotated (45), research remains in the early stages of interpreting their functions. In total, $\sim 18 \%$ of human IncRNAs are considered to be involved in cancer compared with only $\sim 9 \%$ of protein-coding genes (46). Therefore, a systematic study of the molecular mechanisms through which lncRNAs contribute to glioma development and progression will increase the understanding of the disease. In addition, such knowledge may have important implications for improving the early diagnosis, treatment and prognosis of patients with glioma.

NEAT1 expression has been reported to be upregulated in a variety of tumors, and has been proposed as a potential therapeutic target in cancer (47). NEAT1 is regarded as an oncogene in breast cancer, and its downregulation suppresses tumor cell proliferation (48). The results of the present study suggested that NEAT1 may have similarly cancer-promoting effects in glioma. NEAT1 expression was revealed to be upregulated in glioma specimens and cell lines, and to modulate glioma cell proliferation, cell cycle progression and apoptosis by acting as a competitive inhibitor of miR-324-5p binding to KCTD20 mRNA. Additionally, these results were confirmed in vivo using a mouse xenograft model of glioma. Therefore, the present study indicated not only that NEAT1 expression in the tissue may serve as a useful predictor of poor prognosis in glioma, but also that the NEAT1-miR-324-5p-KCTD20 axis may be a potential therapeutic target for the development of novel treatments.

A growing number of studies have suggested that lncRNAs can bind directly to miRNAs, thereby competitively inhibiting sequence-specific interactions with mRNA targets $(49,50)$. In cancer, the lncRNA-mediated sponging of miRNAs could block the tumor suppressor role of specific miRNAs by re-enabling the expression of oncogenic mRNAs. For example, the IncRNA PSMA3 antisense RNA 1 has been demonstrated to promote esophageal cancer cell progression by negatively modulating miR-101 function and thus increasing the expression levels of the proto-oncogene EZH2, a miR-101 target (51). lncRNA urothelial cancer associated 1 functions as an endogenous sponge of miR-182-5p to positively regulate the expression levels of $\delta$-like ligand 4 in renal cancer cells (52). Similarly, NEAT1 has been demonstrated to serve a regulatory role in a variety of cancer types through competitive binding with different miRNAs $(53,54)$. For example, lncRNA NEAT1 regulates NSCLC cell proliferation, migration and invasion by sponging miR-153-3p (53). In addition, lncRNA NEAT1 affects tumor progression by regulating the miR-296-5p/CNN2 axis in hepatocellular carcinoma cells (54). The results of the present study shed light on a similar lncRNA-miRNA-mRNA regulatory mechanism through which NEAT1 promotes glioma cell proliferation. By using a combination of bioinformatics tools, glioma datasets, and in vitro and in vivo assays with glioma cell lines, it was demonstrated that NEAT1 may directly bind to miR-324-5p to exert its regulatory role. These results suggested that miR-324-5p may normally serve an inhibitory role in NHAs, and that its aberrantly low expression in glioma cells may contribute to tumor progression. Since the effects of NEAT1 overexpression were similar to those of miR-324-5p inhibition, it was suggested that NEAT1 may regulate glioma cell proliferation by controlling miR-324-5p expression. In the present study, using glioma cells, the ability of NEAT1 to post-transcriptionally regulate KCTD20 mRNA by acting as a ceRNA for miR-324-5p was demonstrated.

The KCTD protein family consists of 25 members [KCTD1-21, SH3KBP1 binding protein 1, BTB domain containing 10 (BTBD10), TNF $\alpha$ induced protein 1 and potassium channel regulator], which serve a key role in basic physiological and pathological processes $(55,56)$. The KCTD family of proteins have been reported to be involved in a variety of regulatory processes, including $\gamma$-aminobutyric acid type B signaling, proteasome processes, potassium transport and regulation of transcription response (57). Previous studies have demonstrated that these proteins are closely associated with the progression of cancer, including acute myeloid leukemia (58), breast cancer (59) and medulloblastoma (60).

KCTD20, encoded on chromosome 6 , is an isoform of BTBD10 and harbors a C-terminal amino acid sequence similar to that of BTBD10 (61). A recent study demonstrated that KCTD20 is part of a powerful gene network (including the RhoA pathway), involving ubiquitin-related proteasome pathways, protein synthesis/transport and mitotic cell cycle (62). However, to the best of our knowledge, the role of KCTD20 in the development and progression of other types of cancer remains unclear. Consistent with a previous report on KCTD20 in NSCLC, the present study demonstrated that KCTD20 expression was upregulated in glioma tissues compared with in normal tissues (63). The present study demonstrated that miR-324-5p may bind directly to the KCTD20 3'-UTR to regulate its expression. Notably, it was demonstrated that knockdown of KCTD20 in a mouse model of glioma not only reduced tumor growth but also extended mouse survival. In addition, miR-324-5p expression in glioma specimens was inversely correlated with the expression levels of both NEAT1 and KCTD20, while NEAT1 expression was positively correlated with KCTD20 expression. These findings revealed a novel mechanism of the regulation of glioma cell proliferation via the NEAT1-miR-324-5p-KCTD20 axis. The current experimental data revealed that KCTD20 is a novel type of important participant in the growth of glioma, and its downregulation can inhibit cell proliferation in vivo and in vitro. The NEAT1/miR-324-5p/KCTD20 axis may be involved in signal transduction or protein degradation mechanisms, which need to be defined in future studies.

In conclusion, the results of the present study provided mechanistic insights into the function of the lncRNA NEAT1 and demonstrated that it could act as a molecular sponge for miR-324-5p, thereby increasing KCTD20 expression and promoting the proliferation of glioma cells in vitro and in vivo. These findings suggested that NEAT1 may serve as 
a prognostic marker for patients with glioma, and that the NEAT1-miR-324-5p-KCTD20 regulatory axis could be a potential therapeutic target for the development of novel glioma treatments.

\section{Acknowledgements}

Not applicable.

\section{Funding}

The present study was supported by the Natural Science Foundation of China (grant no. 81627806) and the Six Talent Peaks Project in Jiangsu Province [grant no. 2015-WSN-023 (IB15)].

\section{Availability of data and materials}

The datasets used and/or analyzed during the current study are available from the corresponding author on reasonable request.

\section{Authors' contributions}

JZ and YaL performed data analyses and wrote the initial manuscript. JZ, YaL, YuL and GX performed the cell and animal experiments. YH performed the bioinformatics analysis. XL contributed clinical information and samples. JZ, XL and WL designed the study and revised the manuscript. XL and WL confirm the authenticity of all the raw data. All authors read and approved the final manuscript.

\section{Ethics approval and consent to participate}

The present study was approved by the Institutional Review Board and Ethics Committee of the Nanjing Medical University (Nanjing, China) and Fourth Military Medical University (Xi'an, China). All experiments were performed in accordance with the principles of the Declaration of Helsinki and written informed consent was obtained from all patients. All animal experiments were approved by the Animal Management Rule of the Chinese Ministry of Health (document 55, 2001) and were conducted in accordance with the approved guidelines and experimental protocols of the Fourth Military Medical University (Xi'an, China).

\section{Patient consent for publication}

Not applicable.

\section{Competing interests}

The authors declare that they have no competing interests.

\section{References}

1. Ricard D, Idbaih A, Ducray F, Lahutte M, Hoang-Xuan K and Delattre JY: Primary brain tumours in adults. Lancet 379: 1984-1996, 2012.

2. Weller M, Cloughesy T, Perry JR and Wick W: Standards of care for treatment of recurrent glioblastoma-are we there yet? Neuro Oncol 15: 4-27, 2013.
3. Wang J, Su HK, Zhao HF, Chen ZP and To SS: Progress in the application of molecular biomarkers in gliomas. Biochem Biophys Res Commun 465: 1-4, 2015.

4. Stupp R, Mason WP, van den Bent MJ, Weller M, Fisher B, Taphoorn MJ, Belanger $\mathrm{K}$, Brandes AA, Marosi C, Bogdahn U, et al: Radiotherapy plus concomitant and adjuvant temozolomide for glioblastoma. N Engl J Med 352: 987-996, 2005.

5. Shi X, Sun M, Liu H, Yao Y and Song Y: Long non-coding RNAs: A new frontier in the study of human diseases. Cancer Lett 339: 159-166, 2013

6. Ponting CP, Oliver PL and Reik W: Evolution and functions of long noncoding RNAs. Cell 136: 629-641, 2009.

7. Sheik Mohamed J, Gaughwin PM, Lim B, Robson P and Lipovich L: Conserved long noncoding RNAs transcriptionally regulated by Oct 4 and Nanog modulate pluripotency in mouse embryonic stem cells. RNA 16: 324-337, 2010.

8. Yang L, Froberg JE and Lee JT: Long noncoding RNAs: Fresh perspectives into the RNA world. Trends Biochem Sci 39: 35-43, 2014.

9. Guttman M, Donaghey J, Carey BW, Garber M, Grenier JK, Munson G, Young G, Lucas AB, Ach R, Bruhn L, et al: lincRNAs act in the circuitry controlling pluripotency and differentiation. Nature 477: 295-300, 2011.

10. Cai B, Zheng Y, Ma S, Xing Q, Wang X, Yang B, Yin G and Guan F: Long non-coding RNA regulates hair follicle stem cell proliferation and differentiation through PI3K/AKT signal pathway. Mol Med Rep 17: 5477-5483, 2018.

11. Zheng W and Yu A: EZH2-mediated suppression of lncRNA-LET promotes cell apoptosis and inhibits the proliferation of post-burn skin fibroblasts. Int J Mol Med 41: 1949-1957, 2018.

12. Zheng P, Yin Z, Wu Y, Xu Y, Luo Y and Zhang TC: LncRNA HOTAIR promotes cell migration and invasion by regulating MKL1 via inhibition miR206 expression in HeLa cells. Cell Commun Signal 16: 5, 2018.

13. Bueno MJ, Perez de Castro I and Malumbres M: Control of cell proliferation pathways by microRNAs. Cell cycle 7: 3143-3148, 2008.

14. Cesana M, Cacchiarelli D, Legnini I, Santini T, Sthandier O, Chinappi M, Tramontano A and Bozzoni I: A long noncoding RNA controls muscle differentiation by functioning as a competing endogenous RNA. Cell 147: 358-369, 2011.

15. Tsai MC, Manor O, Wan Y, Mosammaparast N, Wang JK, Lan F, Shi Y, Segal E and Chang HY: Long noncoding RNA as modular scaffold of histone modification complexes. Science 329: 689-693, 2010

16. Cech TR and Steitz JA: The noncoding RNA revolution-trashing old rules to forge new ones. Cell 157: 77-94, 2014.

17. Salmena L, Poliseno L, Tay Y, Kats L and Pandolfi PP: A ceRNA hypothesis: The Rosetta Stone of a hidden RNA language? Cell 146: 353-358, 2011.

18. Zhang G, Li S, Lu J, Ge Y, Wang Q, Ma G, Zhao Q, Wu D, Gong W, Du M, et al: LncRNA MT1JP functions as a ceRNA in regulating FBXW7 through competitively binding to miR-92a-3p in gastric cancer. Mol cancer 17: 87, 2018.

19. Zhao L, Zhou Y, Zhao Y, Li Q, Zhou J and Mao Y: Long non-coding RNA TUSC 8 inhibits breast cancer growth and metastasis via miR-190b-5p/MYLIP axis. Aging (Albany NY) 12: 2974-2991, 2020

20. Chen Y, Cheng W, Shi H, Huang S, Chen H, Liu D, Xu W, Yu J and Wang J: Classifying gastric cancer using FLORA reveals clinically relevant molecular subtypes and highlights LINC01614 as a biomarker for patient prognosis. Oncogene 40: 2898-2909, 2021.

21. Zhang Z, Chen F, Zhan H, Chen L, Deng Q, Xiong T, Li Y and Ye J: IncRNA CASC9 sponges miR-758-3p to promote proliferation and EMT in bladder cancer by upregulating TGF- $\beta 2$. Oncol Rep 45: 265-277, 2021.

22. Clemson CM, Hutchinson JN, Sara SA, Ensminger AW, Fox AH, Chess A and Lawrence JB: An architectural role for a nuclear noncoding RNA: NEAT1 RNA is essential for the structure of paraspeckles. Mol Cell 33: 717-726, 2009.

23. Chakravarty D, Sboner A, Nair SS, Giannopoulou E, Li R, Hennig S, Mosquera JM, Pauwels J, Park K, Kossai M, et al: The oestrogen receptor alpha-regulated lncRNA NEAT1 is a critical modulator of prostate cancer. Nat Commun 5: 5383, 2014.

24. Dong P, Xiong Y, Yue J, Xu D, Ihira K, Konno Y, Kobayashi N, Todo $\mathrm{Y}$ and Watari H: Long noncoding RNA NEAT1 drives aggressive endometrial cancer progression via miR-361-regulated networks involving STAT3 and tumor microenvironment-related genes. J Exp Clin Cancer Res 38: 295, 2019. 
25. Dong P, Xiong Y, Yue J, Hanley SJB, Kobayashi N, Todo Y and Watari H: Long Non-coding RNA NEAT1: A novel target for diagnosis and therapy in human tumors. Front Genet 9: 471, 2018.

26. Kong X,Zhao Y, Li X, Tao Z, Hou M and Ma H: Overexpression of HIF-2 $\alpha$-Dependent NEAT1 promotes the progression of non-small cell lung cancer through miR-101-3p/SOX $/ \mathrm{Wnt} / \beta$-catenin signal pathway. Cell Physiol Biochem 52: 368-381, 2019.

27. Huang B, Liu C, Wu Q, Zhang J, Min Q, Sheng T, Wang X and Zou Y: Long non-coding RNA NEAT1 facilitates pancreatic cancer progression through negative modulation of miR-506-3p. Biochem Biophys Res Commun 482: 828-834, 2017.

28. Louis DN, Ohgaki H, Wiestler OD, Cavenee WK, Burger PC, Jouvet A, Scheithauer BW and Kleihues P: The 2007 WHO classification of tumours of the central nervous system. Acta Neuropathol 114: 97-109, 2007.

29. Livak KJ and Schmittgen TD: Analysis of relative gene expression data using real-time quantitative PCR and the 2(-Delta Delta C(T)) Method. Methods 25: 402-408, 2001

30. Peng C, Chen Z, Wang S, Wang HW, Qiu W, Zhao L, Xu R, Luo H, Chen Y, Chen D, et al: The error-prone DNA polymerase $\kappa$ promotes temozolomide resistance in glioblastoma through Rad17-dependent activation of ATR-Chk1 signaling. Cancer Res 76: 2340-2353, 2016.

31. Zhang R, Luo H, Wang S, Chen W, Chen Z, Wang HW, Chen Y, Yang J, Zhang X, Wu W, et al: MicroRNA-377 inhibited proliferation and invasion of human glioblastoma cells by directly targeting specificity protein 1 . Neuro Oncol 16: 1510-1522, 2014

32. http://www.beijing.gov.cn/zhengce/zhengcefagui/qtwj/201912/ t20191219 1304752.html.

33. Cooper LA, Demicco EG, Saltz JH, Powell RT, Rao A and Lazar AJ: PanCancer insights from The Cancer Genome Atlas: The pathologist's perspective. J Pathol 244: 512-524, 2018.

34. Wang Z, Jensen MA and Zenklusen JC: A practical guide to The Cancer Genome Atlas (TCGA). Methods Mol Biol 1418: 111-141, 2016.

35. Walker C, Baborie A, Crooks D, Wilkins S and Jenkinson MD Biology, genetics and imaging of glial cell tumours. $\mathrm{Br}$ J Radiol 84 Spec No 2(Spec Iss 2): S90-106, 2011.

36. Yan W, Zhang W, You G, Zhang J, Han L, Bao Z, Wang Y, Liu Y, Jiang C, Kang C, et al: Molecular classification of gliomas based on whole genome gene expression: A systematic report of 225 samples from the Chinese Glioma Cooperative Group. Neuro Oncol 14: 1432-1440, 2012.

37. Gravendeel LA, Kouwenhoven MC, Gevaert O, de Rooi JJ, Stubbs AP, Duijm JE, Daemen A, Bleeker FE, Bralten LB, Kloosterhof NK, et al: Intrinsic gene expression profiles of gliomas are a better predictor of survival than histology. Cancer Res 69: 9065-9072, 2009.

38. Miao YR, Liu W, Zhang Q and Guo AY: lncRNASNP2: An updated database of functional SNPs and mutations in human and mouse lncRNAs. Nucleic acids Res 46: D276-D280, 2018

39. Chen Y and Wang X: MiRDB: An online database for prediction of functional microRNA targets. Nucleic Acids Res 48 (D1) D127-D131, 2020

40. Shade A and Handelsman J: Beyond the Venn diagram: The hunt for a core microbiome. Environ Microbiol 14: 4-12, 2012.

41. Gutschner T and Diederichs S: The hallmarks of cancer: A long non-coding RNA point of view. RNA Biol 9: 703-719, 2012.

42. Gibb EA, Brown CJ and Lam WL: The functional role of long non-coding RNA in human carcinomas. Mol Cancer 10: 38, 2011

43. Feng Y, Hu X, Zhang Y, Zhang D, Li C and Zhang L: Methods for the study of long noncoding RNA in cancer cell signaling. Methods Mol Biol 1165: 115-143, 2014.

44. Rinn JL and Chang HY: Genome regulation by long noncoding RNAs. Annu Rev Biochem 81: 145-166, 2012.

45. Xie C, Yuan J, Li H, Li M, Zhao G, Bu D, Zhu W, Wu W, Chen R and Zhao Y: NONCODEv4: Exploring the world of long non-coding RNA genes. Nucleic acids Res 42 (Database issue): D98-D103, 2014

46. Khachane AN and Harrison PM: Mining mammalian transcript data for functional long non-coding RNAs. PLoS One 5: e10316, 2010.
47. Yu X, Li Z, Zheng H, Chan MT and Wu WK: NEAT1: A novel cancer-related long non-coding RNA. Cell Prolif 50: e12329, 2017.

48. Shin VY, Chen J, Cheuk IW, Siu MT, Ho CW, Wang X, Jin H and Kwong A: Long non-coding RNA NEAT1 confers oncogenic role in triple-negative breast cancer through modulating chemoresistance and cancer stemness. Cell Death Dis 10: 270, 2019.

49. Yang $\mathrm{C}, \mathrm{Wu} \mathrm{D}$, Gao L, Liu X, Jin Y, Wang D, Wang T and Li X: Competing endogenous RNA networks in human cancer: Hypothesis, validation, and perspectives. Oncotarget 7: 13479-13490, 2016.

50. Jalali S, Bhartiya D, Lalwani MK, Sivasubbu S and Scaria V: Systematic transcriptome wide analysis of lncRNA-miRNA interactions. PLoS One 8: e53823, 2013

51. Qiu BQ, Lin XH, Ye XD, Huang W, Pei X, Xiong D, Long X, Zhu SQ, Lu F, Lin K, et al: Long non-coding RNA PSMA3-AS1 promotes malignant phenotypes of esophageal cancer by modulating the miR-101/EZH2 axis as a ceRNA. Aging (Albany NY) 12: 1843-1856, 2020

52. Wang W, Hu W, Wang Y, An Y, Song L, Shang P and Yue Z: Long non-coding RNA UCA1 promotes malignant phenotypes of renal cancer cells by modulating the miR-182-5p/DLL4 axis as a ceRNA. Mol Cancer 19: 18, 2020.

53. Zhao L, Bi M, Zhang H and Shi M: Downregulation of NEAT1 suppresses cell proliferation, migration, and invasion in NSCLC Via Sponging miR-153-3p. Cancer Biother Radiopharm 35: 362-370, 2020

54. Li Y, Ding X, Xiu S, Du G and Liu Y: IncRNA NEAT1 promotes proliferation, migration and invasion via regulating miR-296-5p/CNN2 axis in hepatocellular carcinoma cells. OncoTargets Ther 12: 9887-9897, 2019.

55. Teng X, Aouacheria A, Lionnard L, Metz KA, Soane L, Kamiya A and Hardwick JM: KCTD: A new gene family involved in neurodevelopmental and neuropsychiatric disorders. CNS Neurosci Ther 25: 887-902, 2019.

56. Liu Z, Xiang Y and Sun G: The KCTD family of proteins: Structure, function, disease relevance. Cell Biosci 3: 45, 2013.

57. Skoblov M, Marakhonov A, Marakasova E, Guskova A, Chandhoke V, Birerdinc A and Baranova A: Protein partners of KCTD proteins provide insights about their functional roles in cell differentiation and vertebrate development. BioEssays 35 : 586-596, 2013

58. Smaldone G, Coppola L, Incoronato M, Parasole R, Ripaldi M, Vitagliano L, Mirabelli P and Salvatore M: KCTD15 protein expression in peripheral blood and acute myeloid leukemia. Diagnostics (Basel) 10: 371, 2020.

59. Rivas J, Díaz N, Silva I, Morales D, Lavanderos B, Álvarez A, Saldías MP, Pulgar E, Cruz P, Maureira D, et al: KCTD5, a novel TRPM4-regulatory protein required for cell migration as a new predictor for breast cancer prognosis. FASEB J 34: 7847-7865, 2020.

60. Canettieri G, Di Marcotullio L, Greco A, Coni S, Antonucci L, Infante P, Pietrosanti L, De Smaele E, Ferretti E, Miele E, et al: Histone deacetylase and Cullin3-REN(KCTD11) ubiquitin ligase interplay regulates Hedgehog signalling through Gli acetylation. Nat Cell Biol 12: 132-142, 2010.

61. Nawa M and Matsuoka M: KCTD20, a relative of BTBD10, is a positive regulator of Akt. BMC Biochem 14: 27, 2013.

62. Huggett SB and Stallings MC: Cocaine'omics: Genome-wide and transcriptome-wide analyses provide biological insight into cocaine use and dependence. Addict Biol 25: e12719, 2020.

63. Zhang X, Zhou H, Cai L, Fan C, Liu Y, Wang L, Li Q and Miao Y: Kctd 20 promotes the development of non-small cell lung cancer through activating Fak/AKT pathway and predicts poor overall survival of patients. Mol Carcinog 56: 2058-2065, 2017.

This work is licensed under a Creative Commons Attribution-NonCommercial-NoDerivatives 4.0 International (CC BY-NC-ND 4.0) License. 